\title{
AUGMENTATIONS AND RULINGS OF LEGENDRIAN KNOTS
}

\author{
C. LEVERSON
}

\begin{abstract}
For any Legendrian $\operatorname{knot} \Lambda$ in $\left(\mathrm{R}^{3}, \operatorname{ker}(d z-y d x)\right)$, we show that the existence of an augmentation to any field of the Chekanov-Eliashberg differential graded algebra over $\mathrm{z}\left[t, t^{-1}\right]$ is equivalent to the existence of a ruling of the front diagram, generalizing results of Fuchs, Ishkhanov, and Sabloff. We also show that any even graded augmentation must send $t$ to -1 .
\end{abstract}

\section{INTRODUCTION}

A Legendrian knot in $\left(\mathbb{R}^{3}, \xi_{\text {std }}\right)$ is an embedding $\Lambda: S^{1} \rightarrow \mathbb{R}^{3}$ which is everywhere tangent to the contact planes. In [4] (see related [6]), Chekanov introduced a combinatorial way to associate a non-commutative differential graded algebra (DGA) over $\mathbb{Z} / 2$ to a Lagrangian diagram of a Legendrian $k n o t ~ \Lambda$ in $\mathbb{R}^{3}$. The DGA is generated by crossings of $\Lambda$ and the differential is determined by a count of immersed polygons whose edges lie on the knot and whose corners lie at crossings of $\Lambda$. In the literature, this DGA is called the ChekanovEliashberg DGA. Chekanov showed that the homology of the DGA is invariant under Legendrian isotopy. He also showed that a linearized version of the homology of the DGA could be used to distinguish between two Legendrian $5_{2}$ knots in $\mathbb{R}^{3}$ which could not be distinguished by the rotation and Thurston-Bennequin numbers. In the early 2000's, Etnyre, Ng, and Sabloff gave a lift of the Chekanov-Eliashberg DGA to a DGA $\left(\mathcal{A}_{R}, \partial\right)$ over $R=\mathbb{Z}\left[t, t^{-1}\right]$ which has a full $\mathbb{Z}$-grading (see [10]). One can recover the Chekanov-Eliashberg DGA by setting $t=1$, which requires one to consider the grading $\bmod 2 r(\Lambda)$, and considering the coefficients mod 2 (where $r(\Lambda)$ is the rotation number, defined in 92 ).

Another Legendrian knot invariant uses generating families, functions whose critical values generate front diagrams of Legendrian knots. Following ideas introduced by Eliashberg in [5, Fuchs [1] and ChekanovPushkar [3] gave invariants involving decompositions of the generating families, which are now called "normal rulings" and can also be used to distinguish between Chekanov's $5_{2}$ knots.

Remarkably, there is a close connection between the Chekanov-Eliashberg DGA and rulings. Fuchs [11, Fuchs-Ishkhanov [12, and Sabloff [17] showed that the existence of a ruling is equivalent to the existence of an augmentation to $\mathbb{Z} / 2$ of the Chekanov-Eliashberg DGA, where an augmentation to a ring $S$ is an algebra map $\epsilon: \mathcal{A}_{R} \rightarrow S$ such that $\epsilon \circ \partial=0$ and $\epsilon(1)=1$.

The main result of this paper gives a generalization of these results using an extension of Sabloff's construction in [17. Let $F$ be a field and $R=\mathbb{Z}\left[t, t^{-1}\right]$. Given a $\rho$-graded augmentation $\epsilon: \mathcal{A}_{R} \rightarrow F$ of the $\mathbb{Z}\left[t, t^{-1}\right]$-differential graded algebra $\left(\mathcal{A}_{R}, \partial\right)$ of a knot $\Lambda$, we will find a $\rho$-graded normal ruling of the knot diagram. Conversely, given a $\rho$-graded normal ruling of the knot diagram, we will define a $\rho$-graded augmentation $\epsilon: \mathcal{A}_{R} \rightarrow F$ of the DGA over $\mathbb{Z}\left[t, t^{-1}\right]$ with $\epsilon(t)=-1$. (For $\rho=0$, this is the so called graded case and for $\rho=1$, the ungraded case.) Terminology will be introduced in 92 ,

In $\$ 3$ and 4 , we will show:

Date: August 20, 2018. 
Theorem 1.1. Let $\Lambda$ be a Legendrian knot in $\mathbb{R}^{3}$. Given a field $F,\left(\mathcal{A}_{R}, \partial\right)$ has a $\rho$-graded augmentation $\epsilon: \mathcal{A}_{R} \rightarrow F$ if and only if any front diagram of $\Lambda$ has a $\rho$-graded normal ruling. Furthermore, if $\rho$ is even, then $\epsilon(t)=-1$.

Note that this generalizes Fuchs, Fuchs-Ishkhanov, and Sabloff's results, giving a correspondence between normal rulings and augmentations to any field $F$ of the DGA over $\mathbb{Z}\left[t, t^{-1}\right]$. This does not contradict the result in [15] that there are augmentations to matrix algebras which do not send $t$ to -1 as the matrix algebras are not fields.

Theorem 1.1 can be extended and interpreted in terms of the augmentation variety for a Legendrian knot. Define

$$
\operatorname{Aug}_{\rho}(\Lambda)=\left\{\epsilon(t): \epsilon \text { a } \rho \text {-graded augmentation of }\left(\mathcal{A}_{R}, \partial\right)\right\} \subset F^{*}
$$

the augmentation variety of $\Lambda$, where $F^{*}=F \backslash\{0\}$.

In higher dimensions, understanding the augmentation variety is interesting and useful (see [1] and [14]), so there has been some question as to whether we can determine the augmentation variety in $\mathbb{R}^{3}$ with the standard contact structure. In $\$ 3$, we prove:

Theorem 1.2. If $\rho$ is odd and $\rho \mid 2 r(\Lambda)$, then

$\operatorname{Aug}_{\rho}(\Lambda)= \begin{cases}\left\{-x^{2}: x \in F^{*}\right\} & \text { if there exists a } \rho \text {-graded normal ruling of } \Lambda \text { which is not oriented (introduced in (3) } \\ \{-1\} & \text { if there exists a } \rho \text {-graded normal ruling of } \Lambda \text { and all rulings are oriented } \\ \varnothing & \text { if there are no } \rho \text {-graded normal rulings of } \Lambda .\end{cases}$

For example, the right handed trefoil $\Lambda$ in Figure 1 has DGA $\left(\mathcal{A}_{R}, \partial\right)$ with $\left|c_{i}\right|=0$ for $1 \leqslant i \leqslant 3$, $\left|c_{4}\right|=\left|c_{5}\right|=1$, and $|t|=0$. Then $\mathcal{A}_{R}=\mathcal{A}_{R}\left(c_{1}, \ldots, c_{5}\right)$ with differential

$$
\begin{aligned}
& \partial c_{1}=\partial c_{2}=\partial c_{3}=0 \\
& \partial c_{4}=t+c_{1}+c_{3}+c_{1} c_{2} c_{3} \\
& \partial c_{5}=1-c_{1}-c_{3}-c_{3} c_{2} c_{1} .
\end{aligned}
$$

Let $F$ be a field. If $\epsilon: \mathcal{A}_{R} \rightarrow F$ is a 1-graded (ungraded) augmentation, then

$$
\begin{aligned}
& 0=\epsilon(t)+\epsilon\left(c_{1}\right)+\epsilon\left(c_{3}\right)+\epsilon\left(c_{1}\right) \epsilon\left(c_{2}\right) \epsilon\left(c_{3}\right) \\
& 0=1-\epsilon\left(c_{1}\right)-\epsilon\left(c_{3}\right)-\epsilon\left(c_{3}\right) \epsilon\left(c_{2}\right) \epsilon\left(c_{1}\right)
\end{aligned}
$$

and so $\epsilon(t)=-1$. Thus $\operatorname{Aug}_{1}(\Lambda)=\{-1\}$.

Now consider the left handed trefoil $\Lambda^{\prime}$ depicted in Figure 1. The associated DGA is $\left(\mathcal{A}_{R}^{\prime}, \partial^{\prime}\right)$ with $\left|c_{1}\right|=\left|c_{2}\right|=\left|c_{4}\right|=-1,\left|c_{3}\right|=\left|c_{5}\right|=\left|c_{6}\right|=1$, and $|t|=2$. Then $\mathcal{A}_{R}=\mathcal{A}_{R}\left(c_{1}, \ldots, c_{6}\right)$ with differential

$$
\begin{aligned}
& \partial^{\prime} c_{1}=\partial^{\prime} c_{2}=\partial^{\prime} c_{3}=0 \\
& \partial^{\prime} c_{4}=t+c_{1} c_{2} \\
& \partial^{\prime} c_{5}=1+c_{2} c_{3} \\
& \partial^{\prime} c_{6}=1+c_{3} c_{1} .
\end{aligned}
$$



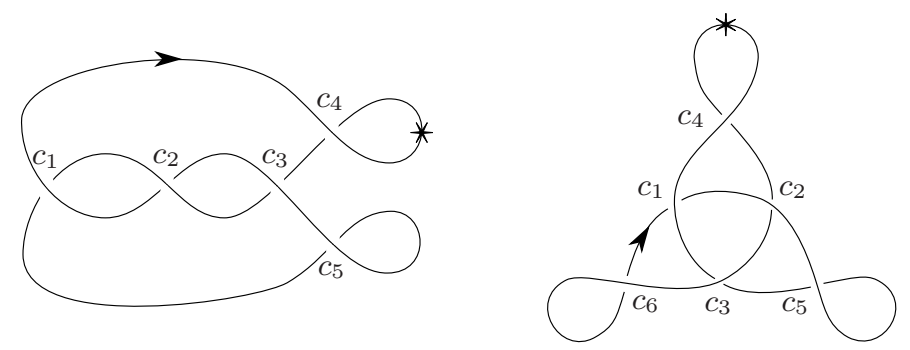

FiguRE 1. The left figure is a Legendrian right handed trefoil and the right is a Legendrian left handed trefoil with crossings labeled. The $*$ indicates the placement of the base point corresponding to $t$.

Let $F$ be a field. If $\epsilon: \mathcal{A}_{R}^{\prime} \rightarrow F$ is a 1 -graded (ungraded) augmentation, then

$$
\begin{aligned}
& 0=\epsilon(t)+\epsilon\left(c_{1}\right) \epsilon\left(c_{2}\right) \\
& 0=1+\epsilon\left(c_{2}\right) \epsilon\left(c_{3}\right) \\
& 0=1+\epsilon\left(c_{3}\right) \epsilon\left(c_{1}\right) .
\end{aligned}
$$

Therefore $\epsilon\left(c_{2}\right)=-\left(\epsilon\left(c_{3}\right)\right)^{-1}=\epsilon\left(c_{1}\right)$ and so $\epsilon(t)=-\left(\epsilon\left(c_{3}\right)\right)^{-2}$. So any nonzero choice of $\epsilon\left(c_{3}\right)$ yields an augmentation and thus $\operatorname{Aug}_{1}\left(\Lambda^{\prime}\right)=\left\{-x^{2}: x \in F^{*}\right\}$.

This result complements the recent work of Henry and Rutherford [13. Henry and Rutherford show that counts of the augmentations to any finite field, without restrictions on where the augmentation sends $t$, are Legendrian knot invariants and that they can be related to the ruling polynomials of the knot, thus showing that the Chekanov-Eliashberg algebra determines the ruling polynomial. Our result shows that if $\rho$ is even, one can restrict the count of $\rho$-graded augmentations to augmentations which send $t$ to -1 , as there are not any which do not.

Theorem 1.1 tells us that if there exists an augmentation to $\mathbb{Z} / 2$, then there exists an augmentation to any field. In 95 , we will show that given an augmentation to $\mathbb{Z} / 2$ of the Chekanov-Eliashberg DGA, we can use constructions similar to those in the proof of Theorem 1.1 to define an augmentation to any ring. In particular:

Theorem 1.3. Let $\Lambda$ be a Legendrian knot in $\mathbb{R}^{3}$. Let $\left(\mathcal{A}_{\mathbb{Z} / 2}, \partial\right)$ be the Chekanov-Eliashberg DGA over $\mathbb{Z} / 2$ and let $\left(\mathcal{A}_{R}, \partial\right)$ be the $D G A$ over $R=\mathbb{Z}\left[t, t^{-1}\right]$. If $\epsilon^{\prime}: \mathcal{A}_{\mathbb{Z} / 2} \rightarrow \mathbb{Z} / 2$ is an augmentation of $\left(\mathcal{A}_{\mathbb{Z} / 2}, \partial\right)$, then one can find a lift of $\epsilon^{\prime}$ to an augmentation $\epsilon: \mathcal{A}_{R} \rightarrow \mathbb{Z}$ of $\left(\mathcal{A}_{R}, \partial\right)$ such that $\epsilon(t)=-1$.

In other words, we will define $\epsilon$ so that the following diagram commutes:

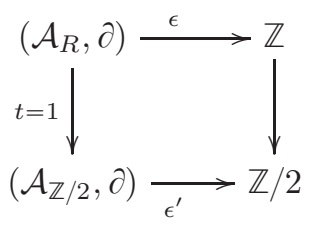

This theorem tells us that given an augmentation to $\mathbb{Z} / 2$ of $\left(\mathcal{A}_{\mathbb{Z} / 2}, \partial\right)$, there exists an augmentation to any ring $S$ of $\left(\mathcal{A}_{R}, \partial\right)$ which sends $t$ to -1 .

1.1. Outline of the article. In $₫ 2$ we recall background on Legendrian knots and give definitions of the Chekanov-Eliashberg DGA, including sign conventions for defining the algebra over $\mathbb{Z}\left[t, t^{-1}\right]$, and a normal ruling. 3 gives the proof that given an augmentation one can define a normal ruling. 4 finishes the proof of Theorem 1.1 by proving that given a normal ruling one can define an augmentation. 44 goes to prove 
Theorem 1.2, giving the augmentation variety in the odd graded case. The paper concludes with the proof of Theorem 1.3 in $\$ 5$.

1.2. Acknowledgements. The author thanks Lenhard Ng for introduction to the problem, for many useful discussions, and for the contribution of the proof of Lemma 3.2. The author also thanks Dan Rutherford for helpful conversations. This work was partially supported by NSF grant DMS-0846346.

\section{Background Material}

2.1. Diagrams of Knots. In this section, we will briefly review necessary ideas of Legendrian knot theory. For further references on this subject, see 8 .

A contact structure on a 3 -manifold $M$ is a completely nonintegrable 2-plane field $\xi$. Locally, a contact structure is the kernel of a 1 -form $\alpha$ which satisfies the non-degeneracy condition

$$
\alpha \wedge d \alpha \neq 0
$$

at every point in $M$. We will be concerned with the standard contact structure on $\mathbb{R}^{3}$, which is the completely nonintegrable 2-plane field $\xi_{0}=\operatorname{ker} \alpha_{0}$, where $\alpha_{0}=d z-y d x$. A Legendrian knot is an embedding $\Lambda: S^{1} \rightarrow \mathbb{R}^{3}$ which is everywhere tangent to the contact planes. A Legendrian isotopy is an ambient isotopy of $\Lambda$ through Legendrian knots. We are interested in Legendrian isotopy classes of Legendrian knots in $\mathbb{R}^{3}$.

The classical invariants for Legendrian isotopy classes of knots are the topological knot type, ThurstonBennequin number, and rotation number (see 2]). The Thurston-Bennequin number measures the self-linking of a Legendrian $\operatorname{knot} \Lambda$. If $\Lambda^{\prime}$ is a knot that is a push off of $\Lambda$ in a direction tangent to the contact structure, then $t b(\Lambda)$ is the linking number of $\Lambda$ and $\Lambda^{\prime}$. The rotation number $r$ of an oriented Legendrian knot $\Lambda$ is the rotation of its tangent vector field with respect to any global trivialization of $\xi_{0}$, for example, $\left\{\partial_{y}, \partial_{x}+y \partial_{z}\right\}$. A natural question is then whether these invariants with the topological knot type alone classify Legendrian knots, in other words, whether all Legendrian knots are "Legendrian simple." Eliashberg and Fraser [7] show that Legendrian unknots are Legendrian simple and Etnyre and Honda [9] show that Legendrian torus and figure eight knots are as well.

Two particularly useful projections of Legendrian knots are the Lagrangian projection and the front projection. The Lagrangian projection is the map

$$
\pi_{\ell}:(x, y, z) \mapsto(x, y) .
$$

The front projection is the map

$$
\pi_{f}:(x, y, z) \mapsto(x, z) .
$$

In general, we will call the Lagrangian projection (resp. front projection) of a Legendrian knot a Lagrangian diagram (resp. front diagram). Figure 2 gives Lagrangian (left) and front (right) projections of a Legendrian version of a right handed trefoil.

Note that one can recover the $y$ coordinate of a knot from the slope of the front diagram (see [8]):

$$
y=\frac{d z}{d x}
$$

This implies that lines tangent to a front diagram of a Legendrian knot are never vertical. Front diagrams instead have semicubical cusps. It also implies that at a double point the strand with the smaller (more negative) slope has a smaller $y$ coordinate and so passes in front of the strand with larger (more positive) 

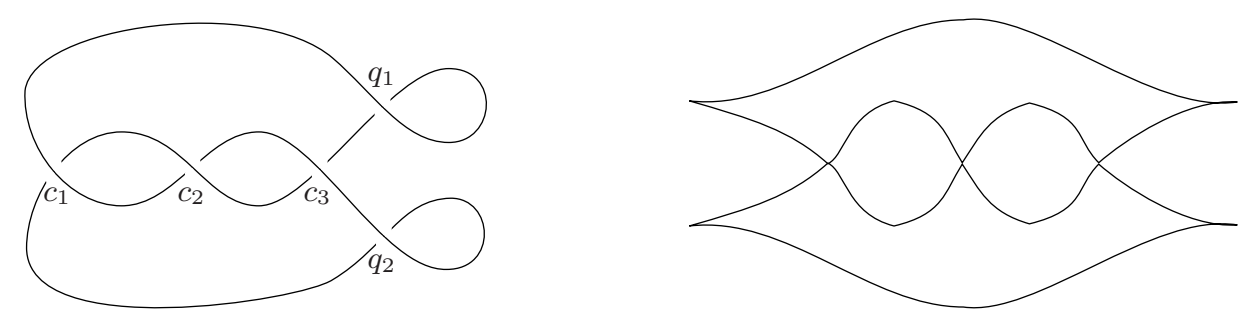

FiguRE 2. The left figure gives a Lagrangian projection of a Legendrian right handed trefoil with crossings labeled and the right figure gives a front projection.

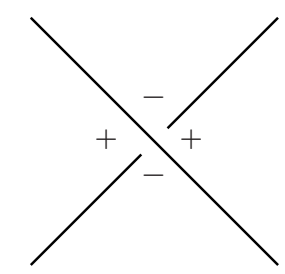

Figure 3. A labeling of the Reeb signs of the quadrants around a crossing.

slope. For a front diagram of an oriented Legendrian knot, the rotation number is half of the difference between the number of downward-pointing cusps and the number of upward-pointing cusps.

In particular, we will find that front diagrams in plat position will be easier to manipulate. A front diagram is in plat position if all of the left cusps have the same $x$ coordinate, all of the right cusps have the same $x$ coordinate, and there do not exist crossings in the diagram which have the same $x$ coordinate. One can use Legendrian versions of the Reidemeister II moves and planar isotopy to put any front diagram into plat position. The diagram of the trefoil given in Figure 2 is an example of a diagram in plat position.

2.2. Definition of the DGA and augmentations. This section contains a brief overview of the differential graded algebra presented by Etnyre, Ng, Sabloff in [10] which lifts the Chekanov-Eliashberg differential graded algebra over $\mathbb{Z} / 2$ in 4 to a DGA over $\mathbb{Z}\left[t, t^{-1}\right]$.

Given a front diagram of an oriented Legendrian $\operatorname{knot} \Lambda$ in plat position in $\mathbb{R}^{3}$ with the standard contact structure, Ng's resolution process [16] gives a Lagrangian diagram for a knot Legendrian isotopic to $\Lambda$ by smoothing left cusps, replacing right cusps with a loop, and resolving crossings so that the over crossing strand has smaller (more negative) slope.

Notation 2.1. Label the crossings of the Lagrangian resolution of a front diagram of $\Lambda$ in plat position by $\left\{c_{1}, \ldots, c_{n}, q_{1}, \ldots, q_{m}\right\}$ with $q_{1}, \ldots, q_{m}$ the crossings from resolving the right cusps labeled from the top to the bottom and $c_{1}, \ldots, c_{n}$ the remaining crossings labeled from left to right (see Figure 6). Label each quadrant around a crossing as shown in Figure 3. We will refer to these labels as the Reeb signs and will call a quadrant at a crossing positive or negative depending on its Reeb sign.

Definition 2.2. Let $\Lambda$ be an oriented Legendrian knot in plat position decorated with $*$ for the base point. The algebra $\mathcal{A}_{R}\left(c_{1}, \ldots, c_{n}, q_{1}, \ldots, q_{m}\right)$ is the noncommutative graded free associative unital algebra over $R=\mathbb{Z}\left[t, t^{-1}\right]$ generated (as an algebra) by $\left\{c_{1}, \ldots, c_{n}, q_{1}, \ldots, q_{m}\right\}$. We will sometimes shorten this to $\mathcal{A}_{R}$.

The grading for $t$ is defined to be $-2 r(\Lambda)$. To give $c_{i}$ a grading, we first must specify a capping path $\gamma_{c_{i}}$. The capping path $\gamma_{c_{i}}$ is the unique path in $\Lambda$ which begins at the under crossing of $c_{i}$, ends at the over 


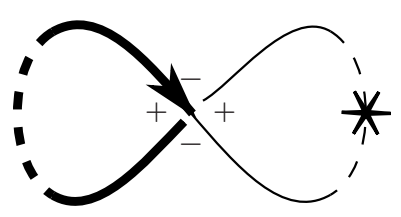

FiguRE 4. The choice of capping path for a crossing, where the capping path is denoted by a heavy line and the arrows give the orientation of the capping path. The signs are the Reeb signs.

crossing of $c_{i}$, and does not go through the base point $*$ (note that this may mean the capping path has the opposite orientation of the knot), as seen in Figure 4

Define the rotation number $r\left(\gamma_{c_{i}}\right)$ to be the fractional number of counterclockwise revolutions made by the tangent vector to $\gamma_{c_{i}}$ as we follow the path. One can perturb the diagram of $\Lambda$ so that all crossings are orthogonal and thus $r\left(\gamma_{c_{i}}\right)$ is an odd multiple of $1 / 4$. Define the grading on $c_{i}$ by

$$
\left|c_{i}\right|=-2 r\left(\gamma_{c_{i}}\right)-\frac{1}{2} \text {. }
$$

(Note that by setting $t=1$ we recover Chekanov's grading from [4, though we then need to consider the grading $\bmod 2 r(\Lambda)$.)

Since we are working with front projections of knots in plat position, we can assign the gradings mod $2 r(\Lambda)$ of crossings at right cusps: $\left|q_{k}\right|=1$. Let $C(\Lambda)$ be the set of points on $\Lambda$ corresponding to cusps of the front projection of $\Lambda$. A Maslov potential function is a locally constant function

$$
\mu: \Lambda \backslash C(\Lambda) \rightarrow \mathbb{Z} / 2 r(\Lambda)
$$

such that for two strands meeting at a cusp (either left or right), the upper strand has Maslov potential one higher than the lower strand. Such a function is well-defined up to a constant. Near a crossing $c_{k}$, let $\alpha_{k}$ be the strand in the front diagram with more negative slope and let $\beta_{k}$ be the strand with more positive slope. The grading defined earlier now becomes

$$
\left|c_{k}\right| \equiv \mu\left(\alpha_{k}\right)-\mu\left(\beta_{k}\right) \quad \bmod 2 r(\Lambda) .
$$

Label a point on the diagram $*$. This will be the base point corresponding to $t$. In $\$ 2.5$ we will discuss the case when we have multiple base points. We define the differential $\partial$ on $\mathcal{A}_{R}\left(c_{1}, \ldots, c_{n}, q_{1}, \ldots, q_{m}\right)$ by appropriately counting embedded disks in the Lagrangian resolution of the front projection of $\Lambda$ in plat position. (Note that, in general, one would need to look for immersed disks, but since $\Lambda$ is in plat position, we need only look for embedded disks.)

Given a generator $a$ and an ordered set of generators $\left\{b_{1}, \ldots, b_{k}\right\}$, let $\Delta\left(a ;\left\{b_{1}, \ldots, b_{k}\right\}\right)$ be the set of orientation-preserving embeddings

$$
f: D^{2} \rightarrow \mathbb{R}^{2}
$$

(up to smooth reparametrization) that map $\partial D^{2}$ to the Lagrangian resolution of $\pi_{f}(\Lambda)$, such that

(1) the restriction of $f$ to $\partial D^{2}$ is an embedding except at $a, b_{1}, \ldots, b_{k}$,

(2) $a, b_{1}, \ldots, b_{k}$ are encountered in counter-clockwise order along $f\left(\partial D^{2}\right)$,

(3) near $a, b_{1}, \ldots, b_{k}, f\left(D^{2}\right)$ covers exactly one quadrant, specifically, a quadrant with positive Reeb sign near $a$ and a quadrant with negative Reeb sign near $b_{i}$ for $1 \leqslant i \leqslant k$.

We can assign a word in $\mathcal{A}$ to each embedded disk by starting with the first corner after the one covering the + quadrant and listing the crossing labels of all negative corners as encountered while following the boundary 

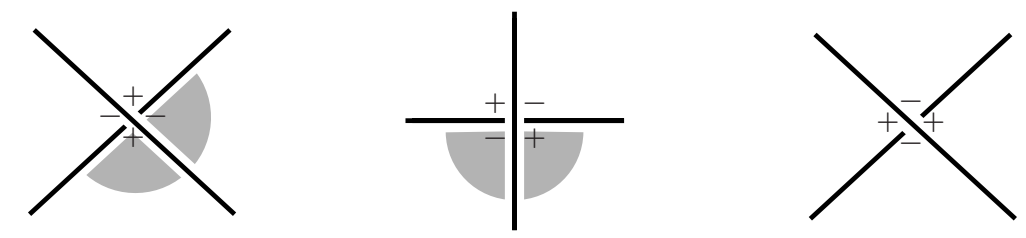

Figure 5. The left two diagrams are positive crossings while the right one is a negative crossing. The signs in the figure are Reeb signs. The orientation signs are -1 for the shaded quadrants and +1 everywhere else. The middle diagram gives the orientation assignment for a positive crossing in a dip, which will be discussed in $\$ 2.4$.

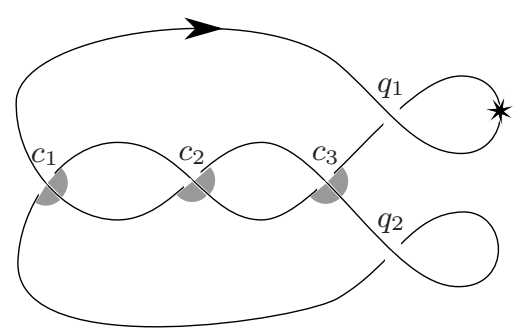

Figure 6. The Lagrangian resolution of the front diagram of the right trefoil in plat position. Crossings are labeled and $*$ indicates the base point corresponding to $t$. The shaded regions are quadrants with orientation sign -1 . All other quadrants have orientation sign +1 .

of the immersed polygon counter-clockwise. We associate a sign to each immersed disk by associating an orientation sign $\epsilon_{Q, a}$ to each quadrant $Q$ in the neighborhood of a crossing $a$, determined by Figure 5 , and defining the sign of a disk $f\left(D^{2}\right)$, the product of the orientation signs over all the corners of the disk, denoted $\epsilon\left(f\left(D^{2}\right)\right)$. Since we are working with a diagram in plat position, in practice, we can define $\epsilon\left(a ; b_{1} \cdots b_{k}\right)$ to be the sign of the unique disk with positive corner at $a$ (with respect to Reeb signs) and negative corners at $b_{1}, \ldots, b_{k}$, the product of the orientation signs over all corners of the disk. Note that our convention for assigning orientation signs differs from [10]. At any crossing $c$ where our convention differs from that in [10], one can recover the convention in [10] by sending $c$ to $-c$.

Define $n_{*}\left(a ; b_{1}, \ldots, b_{k}\right)$ to be the signed count of the number of times one encounters the base point * while following $f\left(\partial D^{2}\right)$ in the counter-clockwise direction, where the sign is determined by whether one encounters the base point while following the orientation of the knot or going against the orientation of the knot.

Definition 2.3. The algebra $\mathcal{A}_{R}$ is a differential graded algebra (DGA) whose differential $\partial$ is defined as follows:

$$
\partial a=\sum_{b_{1}, \ldots, b_{k}} \epsilon\left(a ; b_{1} \cdots b_{k}\right) t^{n_{*}\left(a ; b_{1}, \ldots, b_{k}\right)} b_{1} \cdots b_{k} .
$$

Extend $\partial$ to $\mathcal{A}_{R}$ via $\partial\left(\mathbb{Z}\left[t, t^{-1}\right]\right)=0$ and the signed Leibniz rule:

$$
\partial(v w)=(\partial v) w+(-1)^{|v|} v(\partial w) .
$$

From Theorem 3.7 in [10, the differential $\partial$ has degree -1 and satisfies $\partial^{2}=0$. 
For example, the right handed trefoil depicted in Figure 6 with $r=0$ and $t b=1$ has $\left|c_{i}\right|=0$ and $\left|q_{i}\right|=1$. We have $\mathcal{A}_{R}=\mathcal{A}_{R}\left(c_{1}, c_{2}, c_{3}, q_{1}, q_{2}\right)$ with differential

$$
\begin{aligned}
& \partial c_{1}=\partial c_{2}=\partial c_{3}=0 \\
& \partial q_{1}=t+c_{1}+c_{3}+c_{1} c_{2} c_{3} \\
& \partial q_{2}=1-c_{1}-c_{3}-c_{3} c_{2} c_{1} .
\end{aligned}
$$

Definition 2.4. A graded chain isomorphism

$$
\phi: \mathcal{A}_{R}\left(a_{1}, \ldots, a_{n}\right) \rightarrow \mathcal{A}_{R}\left(b_{1}, \ldots, b_{n}\right)
$$

is elementary if there exists $j \in\{1, \ldots, n\}$ such that

$$
\phi\left(a_{i}\right)= \begin{cases}b_{i} & i \neq j \\ u b_{j}+v & v \in \mathcal{A}_{R}\left(b_{1}, \ldots, b_{j-1}, b_{j+1}, \ldots, b_{n}\right), u \text { a unit in } R, i=j .\end{cases}
$$

A composition of elementary isomorphisms is called tame.

Definition 2.5. Define the algebra $\mathcal{E}_{i}=\mathcal{A}\left(e_{1}^{i}, e_{2}^{i}\right)$ by setting $\left|e_{1}^{i}\right|=i-1,\left|e_{2}^{i}\right|=i, \partial e_{2}^{i}=e_{1}^{i}$, and $\partial e_{1}^{i}=0$.

This algebra models the second Reidemeister move, which produces two new crossings.

Definition 2.6. Given a DGA $\left(\mathcal{A}\left(a_{1}, \ldots, a_{n}\right), \partial\right)$, the degree $i$ stabilization of $\left(\mathcal{A}\left(a_{1}, \ldots, a_{n}\right), \partial\right)$ is defined to be $\mathcal{A}\left(a_{1}, \ldots, a_{n}, e_{1}^{i}, e_{2}^{i}\right)$. The grading and the differential are inherited from $\mathcal{A}$ and $\mathcal{E}_{i}$. Two DGA's $(\mathcal{A}, \partial)$ and $\left(\mathcal{A}^{\prime}, \partial^{\prime}\right)$ are stable tame isomorphic if there exist two sequences of stabilizations $S_{i_{1}}, \ldots, S_{i_{n}}$ and $S_{j_{1}}, \ldots, S_{j_{m}}$ and a tame isomorphism

$$
\phi: S_{i_{n}}\left(\cdots\left(S_{i_{1}}(\mathcal{A})\right) \cdots\right) \rightarrow S_{j_{m}}\left(\cdots\left(S_{j_{1}}\left(\mathcal{A}^{\prime}\right)\right) \cdots\right),
$$

which is also a chain map.

In fact, the stable tame isomorphism class of the DGA is invariant under Legendrian isotopy. Chekanov proved this result over $\mathbb{Z} / 2$ in 4 ] and Etnyre, $\mathrm{Ng}$, and Sabloff proved this result over $\mathbb{Z}\left[t, t^{-1}\right]$ in $[10$.

Now that we have the DGA associated with the projection of $\Lambda$, we can discuss the augmentations.

Definition 2.7. Let $F$ be a field. An augmentation of $\left(\mathcal{A}_{R}, \partial\right)$ to $F$ is an algebra map $\epsilon: \mathcal{A}_{R} \rightarrow F$ such that $\epsilon \circ \partial=0$ and $\epsilon(1)=1$. If $\rho \mid 2 r(\Lambda)$ and $\epsilon$ is supported on generators of degree divisible by $\rho$, then $\epsilon$ is $\rho$-graded. In particular, if $\rho=0$, we say it is graded and if $\rho=1$, we say it is ungraded. We call a generator $a$ augmented if $\epsilon(a) \neq 0$.

For example, if we recall the DGA over $\mathbb{Z}\left[t, t^{-1}\right]$ for the right handed trefoil, then we can classify the augmentations to any field $F$ as follows: Let $\epsilon: \mathcal{A}_{R} \rightarrow F$ be an augmentation. Then $\epsilon(t)=-1$ and

- if $\epsilon\left(c_{1}\right)=0$, then $\epsilon\left(c_{3}\right)=1$ and $\epsilon\left(c_{2}\right) \in F$

- if $\epsilon\left(c_{3}\right)=0$, then $\epsilon\left(c_{1}\right)=1$ and $\epsilon\left(c_{2}\right) \in F$

- if $\epsilon\left(c_{1}\right), \epsilon\left(c_{3}\right) \neq 0$, then

$$
\epsilon\left(c_{2}\right)=\left(1-\epsilon\left(c_{1}\right)-\epsilon\left(c_{3}\right)\right)\left(\epsilon\left(c_{1}\right)\right)^{-1}\left(\epsilon\left(c_{3}\right)\right)^{-1} .
$$

Note that if $F$ is a finite field, as in [13, and $|F|$ is the number of elements in $F$, then we see that there are $|F|$ augmentations of the first type, $|F|$ augmentations of the second type, and $\left|F^{*}\right|^{2}$ augmentations of the 
third type, where $F^{*}=F \backslash\{0\}$. In fact,

$$
\left\{\left(\epsilon\left(c_{1}\right), \epsilon\left(c_{2}\right), \epsilon\left(c_{3}\right), \epsilon\left(q_{1}\right), \epsilon\left(q_{2}\right), \epsilon(t)\right): \epsilon \text { an augmentation to } F\right\}=F \coprod F \coprod\left(F^{*}\right)^{2} .
$$

In [13, this is called the augmentation variety of $(\mathcal{A}(\Lambda), \partial)$. Comparing this with possible rulings of the trefoil, definition given in 2.3 , one sees that (10) coincides with Theorem 3.4 of [13].

For example, the following are examples of graded augmentations to $\mathbb{R}$.

\begin{tabular}{c|cccccc} 
& $c_{1}$ & $c_{2}$ & $c_{3}$ & $q_{1}$ & $q_{2}$ & $t$ \\
\hline$\epsilon_{1}$ & 1 & $\frac{1}{2}$ & 0 & 0 & 0 & -1 \\
$\epsilon_{2}$ & 0 & $\frac{1}{2}$ & 1 & 0 & 0 & -1 \\
$\epsilon_{3}$ & 2 & $\frac{3}{4}$ & $-\frac{2}{5}$ & 0 & 0 & -1 \\
$\epsilon_{4}$ & $-\frac{2}{5}$ & $\frac{3}{4}$ & 2 & 0 & 0 & -1 \\
$\epsilon_{5}$ & $\frac{1}{2}$ & 0 & $\frac{1}{2}$ & 0 & 0 & -1
\end{tabular}

Note that any augmentation of a stabilization $S(\mathcal{A})$ restricts to an augmentation of the smaller algebra $\mathcal{A}$ and any augmentation of the algebra $\mathcal{A}$ extends to an augmentation of the stabilization $S(\mathcal{A})$ where the augmentation sends $e_{1}^{i}$ to 0 and $e_{2}^{i}$ to an arbitrary element of $F$ if $\rho \mid i$ and 0 otherwise.

2.3. Rulings. This paper will show that there is a way to construct an augmentation from a normal ruling and a normal ruling from an augmentation.

Definition 2.8. Consider a front diagram in plat position of a Legendrian knot $\Lambda$. A ruling of this diagram consists of a one-to-one correspondence between the set of left cusps and the set of right cusps where, for each pair of corresponding cusps, two paths in the front diagram join them. These ruling paths must satisfy the following:

(1) Any two paths in the ruling only meet at crossings or cusps;

(2) The interiors of the two paths joining corresponding cusps are disjoint. Thus each pair of paths bound a topological disk.

The first condition tells us the ruling paths never overlap at more than a finite number of points. The second condition tells us that there are disks similar to those in the differential $\partial$, but possibly with "obtuse" corners. As noted in [11, these imply that the ruling paths cover the front diagram and the $x$-coordinate of each path in the ruling is monotonic.

Near a crossing, the two ruling paths which intersect at the crossing are called crossing paths. The two paths paired with the crossing paths are called companion paths.

Given a ruling, at any crossing, we either have that the crossing paths pass through each other, or one path lies entirely above (has $z$-coordinate strictly greater than) the other. In the latter case, we say the ruling is switched at the crossing. If all of the switched crossings in the ruling are of the form (a), (b), or (c), as seen in Figure 7 then we say the ruling is normal. Thus, the possible configurations near a crossing in a normal ruling are shown in Figure 7.

If all of the switched crossings have grading divisible by $\rho$ for some $\rho$ such that $\rho \mid 2 r(\Lambda)$, then we say the ruling is $\rho$-graded. In particular, if $\rho=0$, then we say the ruling is graded and if $\rho=1$, then we say the ruling is ungraded.

For example, if $F=\mathbb{Z} / 2 \mathbb{Z}$, the trefoil has three graded normal rulings as seen in Figure 8 


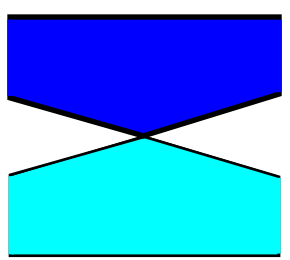

(a)

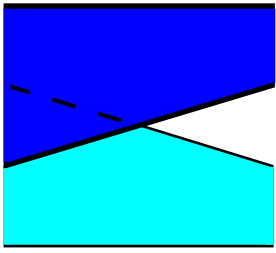

$(d)$

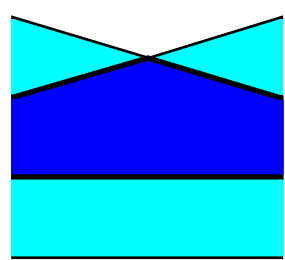

(b)

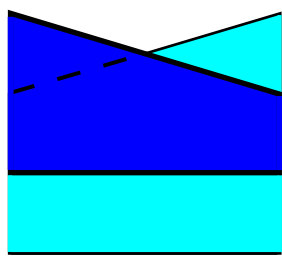

(e)

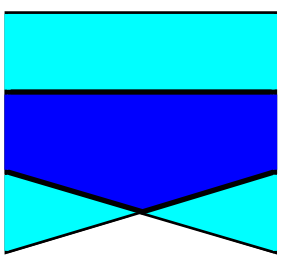

(c)

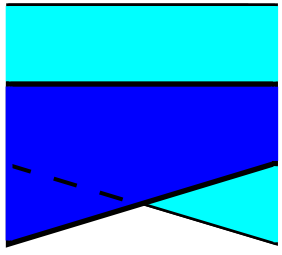

$(f)$

FIGURE 7. By including vertical reflections of (d), (e), and (f), these are all possible configurations of crossings appearing in a normal ruling. The top row contains all possible configurations for switched crossings in a normal ruling.
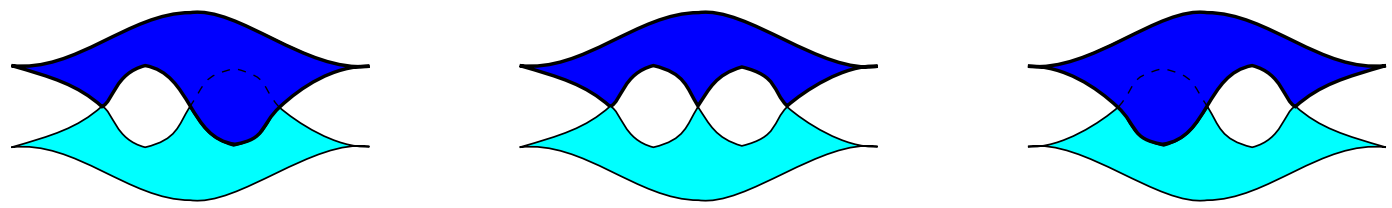

FiguRE 8. The graded normal rulings of the right handed trefoil.

In [3, Chekanov showed that the number of $\rho$-graded normal rulings is invariant under Legendrian isotopy.

2.4. Dips. We will construct a normal ruling of the diagram by using the augmentation to construct an augmentation $\epsilon$ of the dipped diagram satisfying Property (R), as called in [17]. However, the notation in the following section will be necessary to write down Property $(\mathrm{R})$.

Given a Legendrian knot $\Lambda$ in plat position, we construct a dip between two crossings by a sequence of Reidemeister II moves, as seen in Figure 9 in the front projection and Lagrangian projection. In the front projection, it is clear that the diagram with the dip is isotopic to the original diagram. To construct a dip, number the $2 \mathrm{~m}$ strands from bottom to top. Using a type II Reidemeister move, push strand 2 over strand 1 , then strand 3 over strand 1 , then strand 3 over strand 2 , and so on. So that strand $k$ is pushed over strand $\ell$ in lexicographic order. If strand $k$ crosses strand $\ell$ after strand $i$ crosses strand $j$, we write $(i, j)<(k, \ell)$.

The dipped diagram involves introducing a dip between each crossing in the plat position diagram and between the left, respectively right, cusps and the first, respectively last, crossing (see Figure 13). Each Reidemeister II move introduces two new variables. For the dip immediately after crossing $c_{k}$, we will use $a_{r s}^{k}$ and $b_{r s}^{k}$ to denote the new crossings introduced when strand $r$ is passed over strand $s(r>s)$, with $b_{r s}^{k}$ being the leftmost and $a_{r s}^{k}$ being the rightmost new crossing (see Figure 9). We will say the $b_{r s}^{k}$ generators belong to the $b^{k}$-lattice and the $a_{r s}^{k}$ belong to the $a^{k}$-lattice. Thus we will have $a^{k} / b^{k}$-lattices for $0 \leqslant k \leqslant n$. 


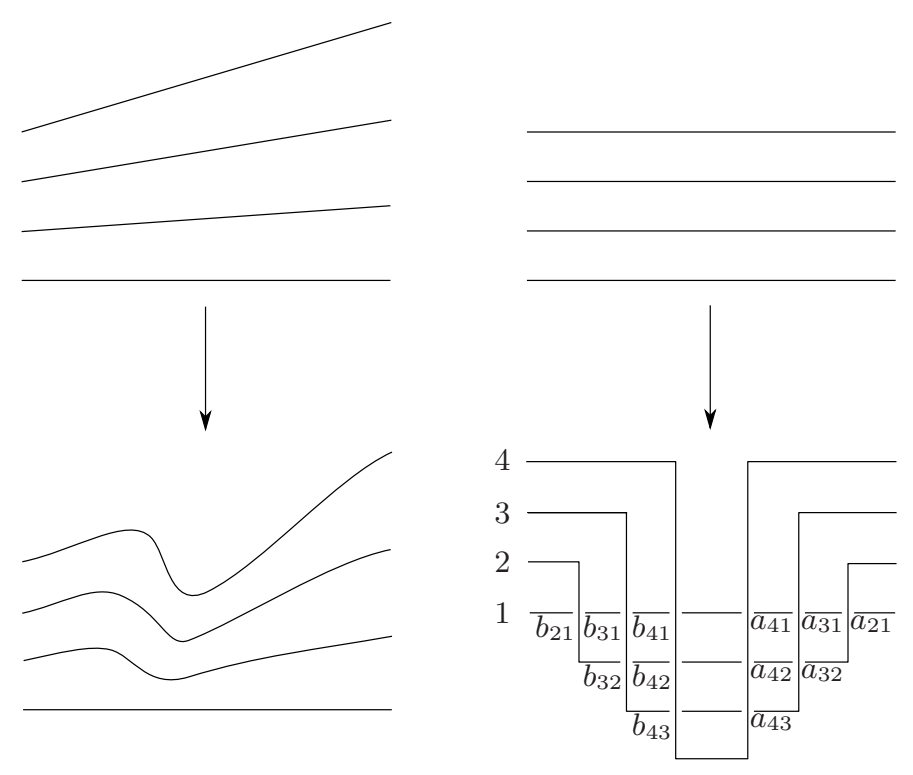

Figure 9. The left diagram gives the modification of the front projection when creating a dip, while the right diagram gives the modification of the Lagrangian projection. In the Lagrangian projection, the $b^{k}$-lattice is made up of the crossings on the left and the $a^{k}$ lattice is made up of the crossings on the right. The crossings in the $b^{k}$-lattice are labeled down and to the left, while the crossings in the $a^{k}$-lattice to the right, with $k$ 's suppressed.

While dipped diagrams have many more crossings than the original knot diagram, the differential $\partial$ on $\mathcal{A}_{R}$ is generally much simpler. We note that if $\mu$ is a Maslov potential function on the front diagram, then

$$
\left|b_{r s}^{k}\right|=\mu(r)-\mu(s) \text {. }
$$

Since the differential $\partial$ lowers degree by one,

$$
\left|a_{r s}^{k}\right|=\left|b_{r s}^{k}\right|-1 .
$$

Orientation sign assignments are given in Figure 5. We can reduce possible disks, and thus possible terms in the differential, further in certain cases. As the disks in the computation of $\mathcal{A}_{\mathbb{Z} / 2}$ are the same disks in the computation of $\mathcal{A}_{R}$, we have the following lemma from [17].

Lemma 2.9 (17] Lemma 3.1). If a and $b$ are the new crossings created by a type II move during the creation of a dip and $y$ is any other crossing, then a appears at most once in any term of $\partial y$, and if a appears in any term of $\partial y$, then $b$ does not.

This follows from considering the disks which have a negative corner at $a$ as seen in Figure 10.

Through consideration of the dipped diagram, we see

- the differential of crossings in the $b^{k}$-lattice involve at most

$-c_{k}$

- base points (we will discuss the case when we have more than one in the next section),

- crossings in the $a^{k-1}$-lattice,

- crossings in the $a^{k}$-lattice,

- the differential of crossings in the $a^{k}$-lattice only involve

- base points,

- crossings in the $b^{k}$-lattice, 

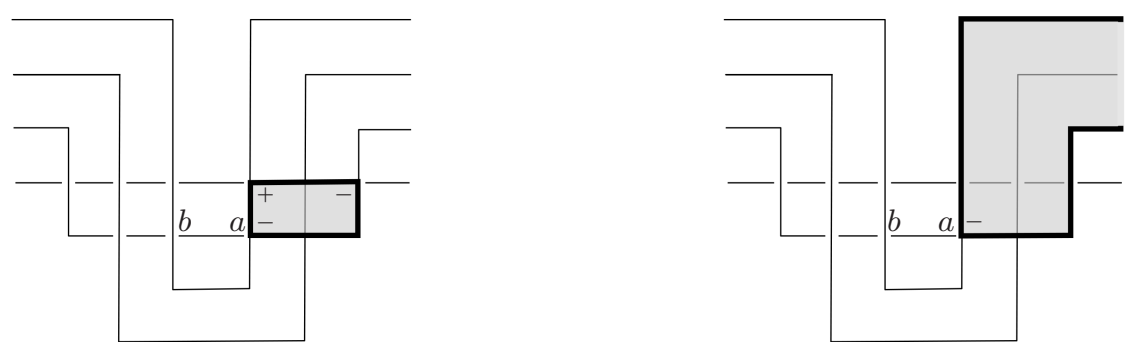

Figure 10. Possible disks contributing to $\partial$ with a negative corner at $a$.

- the differential of $c_{k}$ is 0

for all $1 \leqslant k \leqslant n$. This greatly reduces the types of totally augmented disks for which to look to compute whether we have an augmentation, where a totally augmented disk is a disk which contributes to the differential, all of whose negative corners are augmented.

Notation 2.10. $a_{\{r, s\}}^{k}=a_{\max (r, s), \min (r, s)}^{k}$

2.5. Augmentations before and after a base point move. As we create dips, we will find that the signs are simpler if, in certain cases, we add in a few extra base points. In [15], $\mathrm{Ng}$ and Rutherford give the DGA isomorphisms induced by adding a base point and by moving one base point around a knot. First, we need to extend our definition of the DGA over $\mathbb{Z}\left[t, t^{-1}\right]$ to a DGA over $\mathbb{Z}\left[t_{1}^{ \pm 1}, \ldots, t_{s}^{ \pm 1}\right]$, which we will call $\mathcal{A}(\Lambda, *)$. To this end, label $s$ points on the Lagrangian resolution of the front diagram of $\Lambda$ by the base points $*_{1}, \ldots, *_{s}$ respectively associated to $t_{1}, \ldots, t_{s}$.

Definition 2.11. The algebra $\mathcal{A}$ is a DGA whose grading is defined analogously to the case when there is only one base point: We define $\left|t_{1}\right|=-2 r(\Lambda)$ and $\left|t_{i}\right|=0$ for $1<i \leqslant s$. Given a crossing $c$, let $\gamma_{c}$ be the unique path following the under strand of $c$ to the over strand of $c$ while avoiding $*_{1}$ and define $|c|=-2 r\left(\gamma_{c}\right)-\frac{1}{2}$. The differential $\partial$ is defined as follows:

$$
\partial a=\sum_{b_{1}, \ldots, b_{k}} \epsilon\left(a ; b_{1} \cdots b_{k}\right) t_{1}^{n_{*_{1}}\left(a ; b_{1}, \ldots, b_{k}\right)} \cdots t_{s}^{n_{*_{s}}\left(a ; b_{1}, \ldots, b_{k}\right)} b_{1} \cdots b_{k} .
$$

Extend $\partial$ to $\mathcal{A}$ via $\partial\left(\mathbb{Z}\left[t, t^{-1}\right]\right)=0$ and the signed Leibniz rule:

$$
\partial(v w)=(\partial v) w+(-1)^{|v|} v(\partial w) .
$$

Theorem 2.12 ([15] Thm 2.19). The map $\partial: \mathcal{A}(\Lambda, *) \rightarrow \mathcal{A}(\Lambda, *)$ lowers degree by 1 and is a differential: $\partial^{2}=0$. Up to stable tame isomorphism, the differential graded algebra $(\mathcal{A}(\Lambda, *), \partial)$ is an invariant of $\Lambda$ under Legendrian isotopy (and choice of base point).

Theorem $2.13\left([15]\right.$ Thm 2.20). Let $*_{1}, \ldots, *_{k}$ and $*_{1}^{\prime}, \ldots, *_{k}^{\prime}$ denote two collections of base points on the Lagrangian resolution of the front diagram of a Legendrian knot $\Lambda$, each of which is cyclically ordered along $\Lambda$. Let $\left(\mathcal{A}\left(\Lambda, *_{1}, \ldots, *_{k}\right), \partial\right)$ and $\left.\mathcal{A}\left(\Lambda, *_{1}^{\prime}, \ldots, *_{k}^{\prime}\right), \partial^{\prime}\right)$ denote the corresponding multi-pointed DGAs. Then there is a DGA isomorphism $\Psi:\left(\mathcal{A}\left(\Lambda, *_{1}, \ldots, *_{k}\right), \partial\right) \rightarrow\left(\mathcal{A}\left(\Lambda, *_{1}^{\prime}, \ldots, *_{k}^{\prime}\right), \partial^{\prime}\right)$ such that $\Psi\left(t_{i}\right)=t_{i}$ for all $i$.

In the proof of this theorem, the isomorphism $\Psi$ is defined so that $\Psi\left(c_{j}\right)=c_{j}$ if no base point is pushed over or under the crossing $c_{j}$. If, however, the base point $*_{i}$ is pushed over crossing $c_{j}$, then $\Psi\left(c_{j}\right)=t_{i}^{ \pm 1} c_{j}$, the sign depending on whether the base point is pushed along the knot in the direction of the orientation or 

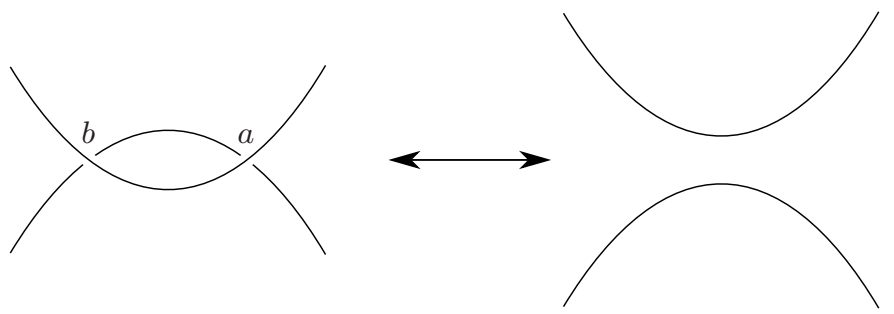

Figure 11. A type II Reidemeister move with crossings $a$ and $b$.

against the orientation of the knot. If the base point $*_{i}$ is pushed under the $\operatorname{crossing} c_{j}$, then $\Psi\left(c_{j}\right)=c_{j} t_{i}^{ \pm 1}$, again, the sign depending on the orientation of the knot.

Theorem 2.14 (15] Thm 2.21). Let $*_{1}, \ldots, *_{k}$ be a cyclically ordered collection of base points along $\Lambda$, and let $*$ be a single base point on $\Lambda$. Then there is a DGA homomorphism $\phi:(\mathcal{A}(\Lambda, *), \partial) \rightarrow\left(\mathcal{A}\left(\Lambda, *_{1}, \ldots, *_{k}\right), \partial\right)$ such that $\phi \circ \partial=\partial \circ \phi$ and $\phi(t)=t_{1} \cdots t_{k}$.

Thus, we can assume there is one base point on each of the right cusps. Also, this shows us that if $\epsilon^{\prime}$ is an augmentation on the diagram after moving the base point $*_{i}$ over the crossing $c_{j}$, then $\epsilon=\epsilon^{\prime} \Psi$ is an augmentation on the diagram before moving the base point.

Remark 2.15. In summary, if $\epsilon\left(t_{i}\right)=-1$, then moving the base point $*_{i}$ over or under a crossing only changes the augmentation by changing the sign of the augmentation on that crossing, no matter the orientation of the strand.

Note that these theorems tell us that if $t$ is the variable associated to the original base point $*$, and $t_{1}, \ldots, t_{s}$ are the variables associated to the base points $*_{1}, \ldots, *_{s}$ in the new diagram, $\epsilon^{\prime}$ is an augmentation on the original diagram, and $\epsilon$ is augmentation on the new diagram resulting from Theorem 2.14, then

$$
\epsilon^{\prime}(t)=\epsilon\left(t_{1} \cdots t_{s}\right)=\prod_{i=1}^{s} \epsilon\left(t_{i}\right)
$$

2.6. Augmentations before and after type II moves. To understand how augmentations before the addition of a dip relate to augmentations after, we need to consider the stable DGA isomorphism induced by a type II move. Suppose $\left(\mathcal{A}_{Z}^{\prime}, \partial^{\prime}\right)$ is the DGA over $Z$ for a knot diagram before a type II move and that $\left(\mathcal{A}_{Z}, \partial\right)$ is the DGA over $Z$ afterward. So

$$
\begin{aligned}
& \mathcal{A}_{Z}=\mathcal{A}_{Z}\left(a, b, a_{1}, \ldots, a_{r}, b_{1}, \ldots, b_{s} ; \partial\right) \\
& \mathcal{A}_{Z}^{\prime}=\mathcal{A}_{Z}\left(a_{1}, \ldots, a_{r}, b_{1}, \ldots, b_{s} ; \partial^{\prime}\right),
\end{aligned}
$$

where $Z=\mathbb{Z}\left[t_{1}, t_{1}^{-1}, \ldots, t_{q}, t_{q}^{-1}\right]$. Suppose that the other crossings are ordered by height:

$$
h\left(b_{s}\right) \geqslant \cdots \geqslant h\left(b_{1}\right) \geqslant h(b)>h(a) \geqslant h\left(a_{1}\right) \geqslant \cdots \geqslant h\left(a_{r}\right) .
$$

It is possible to construct a dip in the plat diagram so that this ordering takes the following form: Suppose strand $k$ is pushed over strand $\ell$. Each $a_{j}$ either lies to the left of the dip or $a_{j}=a_{m n}$ or $b_{m n}$ with $m-n \leqslant k-\ell$. Similarly, $b_{j}$ either lies to the right of the dip or $b_{j}=a_{m n}$ or $b_{m n}$ with $m-n>k-\ell$.

Recall the algebra $\mathcal{E}_{i}=\mathcal{A}_{Z}\left(e_{1}, e_{2}\right)$ with $\left|e_{1}\right|=i-1,\left|e_{2}\right|=i, \partial e_{2}=e_{1}$, and $\partial e_{1}=0$. Define the vector space map $H: S\left(\mathcal{A}_{Z}^{\prime}\right) \rightarrow S\left(\mathcal{A}_{Z}^{\prime}\right)$ by 


$$
H(w)= \begin{cases}0 & w \in \mathcal{A}_{Z}^{\prime} \\ 0 & w=Q e_{2} R \text { with } Q \in \mathcal{A}_{Z}^{\prime}, R \in S\left(\mathcal{A}_{Z}^{\prime}\right) \\ (-1)^{|Q|+1} Q e_{2} R & w=Q e_{1} R \text { with } Q \in \mathcal{A}_{Z}^{\prime}, R \in S\left(\mathcal{A}_{Z}^{\prime}\right) .\end{cases}
$$

Note that either crossing $a$ or $b$ is a positive crossing, so $\partial b=-a+v$, where $v$ is a sum of terms in the $a_{i}$ and $t_{i}^{ \pm 1}$. Define $\Phi_{0}: \mathcal{A}_{Z} \rightarrow S_{|b|}\left(\mathcal{A}_{Z}^{\prime}\right)$ by

$$
\Phi_{0}(w)= \begin{cases}e_{2} & w=b \\ -e_{1}+v & w=a \\ w & \text { otherwise. }\end{cases}
$$

[10] tells us $\Phi_{0}$ is a grading-preserving elementary isomorphism. Inductively define maps $\Phi_{i}$ on the generators of $\mathcal{A}_{Z}$ by:

$$
\Phi_{i}(w)= \begin{cases}b_{i}+H\left(\partial^{\prime} b_{i}-\Phi_{i-1} \partial b_{i}\right) & w=b_{i} \\ \Phi_{i-1}(w) & \text { otherwise. }\end{cases}
$$

In [10, it is shown that $\Phi:=\Phi_{s}$ is a DGA isomorphism between $\mathcal{A}_{Z}$ and $S_{|b|}\left(\mathcal{A}_{Z}^{\prime}\right)$.

If there is an augmentation $\epsilon^{\prime}$ on $S\left(\mathcal{A}_{Z}^{\prime}\right)$, then $\epsilon=\epsilon^{\prime} \Phi$ is an augmentation on $\mathcal{A}_{Z}$. One can check that

$$
\epsilon\left(a_{i}\right)=\epsilon^{\prime}\left(a_{i}\right), \quad \epsilon(a)=\epsilon^{\prime}(v), \quad \epsilon(b)=\epsilon^{\prime}\left(e_{2}\right) .
$$

Recall that if $\left|e_{2}\right|=0$, then $\epsilon^{\prime}\left(e_{2}\right)$ can be chosen arbitrarily.

Analogous to the result for the $\mathbb{Z} / 2$ case in [17, we have:

Lemma 2.16. After a type II Reidemeister move involved in making a dip in a plat diagram, suppose $\epsilon\left(b_{i}\right)$ has been determined for $i<j$. Then

$$
\epsilon\left(b_{j}\right)=\epsilon^{\prime}\left(b_{j}\right)-\sum_{p} \epsilon\left(b_{j} ; Q_{p} a R_{p}\right)(-1)^{\left|\Phi\left(Q_{p}\right)\right|} \epsilon\left(Q_{p} b R_{p}\right)
$$

for $Q_{p}, R_{p} \in \mathcal{A}_{Z}^{\prime}$ such that $\partial b_{j}=P+\sum_{p} \epsilon\left(b ; Q_{p} a R_{p}\right) Q_{p} a R_{p}$ where $P$ is the sum of the terms in $\partial b_{j}$ which do not contain a.

Proof. We know

$$
\Phi\left(b_{i}\right)=b_{i}+H\left(\partial^{\prime} b_{i}-\Phi \partial b_{i}\right) .
$$

We will prove the result by inducting on $j$. For the base case, suppose $j=1$. Since $\partial$ lowers height, we know $\partial b_{1} \in \mathcal{A}_{Z}\left(a, b, a_{1}, \ldots, a_{r}\right)$ and $\partial^{\prime} b_{1} \in \mathcal{A}_{Z}\left(a_{1}, \ldots, a_{r}\right)$. By Lemma 2.9 we know if $P$ is the sum of terms in $\partial b_{1}$ which do not contain $a$, then $\partial b_{1}$ has the form

$$
\partial b_{1}=P+\sum_{p} \epsilon\left(b_{1} ; Q_{p} a R_{p}\right) Q_{p} a R_{p},
$$

where $Q_{p}, R_{p} \in \mathcal{A}_{Z}\left(a_{1}, \ldots, a_{r}\right)$. Therefore

$$
\begin{aligned}
H\left(\partial^{\prime} b_{1}-\Phi \partial b_{1}\right) & =H\left(\partial^{\prime} b_{1}-\Phi\left(P+\sum_{p} \epsilon\left(b_{1} ; Q_{p} a R_{p}\right) Q_{p} a R_{p}\right)\right) \\
& =H\left(\partial^{\prime} b_{1}-\Phi(P)-\sum_{p} \epsilon\left(b_{1} ; Q_{p} a R_{p}\right) Q_{p}\left(-e_{1}+v\right) R_{p}\right) .
\end{aligned}
$$


We know $\partial^{\prime} b_{1} \in \mathcal{A}_{Z}\left(a_{1}, \ldots, a_{r}\right)$, so $H\left(\partial^{\prime} b_{1}\right)=0$. Since $P \in \mathcal{A}_{Z}\left(b, a_{1}, \ldots, a_{r}\right)$, we know $\Phi(P) \in \mathcal{A}_{Z}\left(e_{2}, a_{1}, \ldots, a_{r}\right)$ and so $H(\Phi(P))=0$. Thus

$$
\begin{aligned}
H\left(\partial^{\prime} b_{1}-\Phi \partial b_{1}\right) & =-\sum_{p} \epsilon\left(b_{1} ; Q_{p} a R_{p}\right) H\left(Q_{p}\left(-e_{1}+v\right) R_{p}\right) \\
& =\sum_{p}(-1)^{\left|Q_{p}\right|+1} \epsilon\left(b_{1} ; Q_{p} a R_{p}\right) Q_{p} e_{2} R_{p} .
\end{aligned}
$$

So

$$
\begin{aligned}
\epsilon\left(b_{1}\right) & =\epsilon^{\prime}\left(\Phi\left(b_{1}\right)\right) \\
& =\epsilon^{\prime}\left(b_{1}+H\left(\partial^{\prime} b_{1}-\Phi \partial b_{1}\right)\right) \\
& =\epsilon^{\prime}\left(b_{1}\right)+\epsilon^{\prime}\left(\sum_{p}(-1)^{\left|Q_{p}\right|+1} \epsilon\left(b_{1} ; Q_{p} a R_{p}\right) Q_{p} e_{2} R_{p}\right) \\
& =\epsilon^{\prime}\left(b_{1}\right)-\sum_{p}(-1)^{\left|Q_{p}\right|} \epsilon\left(b_{1} ; Q_{p} a R_{p}\right) \epsilon\left(Q_{p} b R_{p}\right) .
\end{aligned}
$$

Since

$$
\begin{aligned}
\Phi\left(b_{1}\right) & =b_{1}+H\left(\partial^{\prime} b_{1}-\Phi \partial b_{1}\right) \\
& =b_{1}-\sum_{p}(-1)^{\left|Q_{p}\right|} \epsilon\left(b_{1} ; Q_{p} a R_{p}\right) Q_{p} e_{2} R_{p},
\end{aligned}
$$

we have also shown that $e_{1}$ does not appear in $\Phi\left(b_{1}\right)$.

Now suppose the equation is satisfied for $b_{i}$ and that $e_{1}$ does not appear in $\Phi\left(b_{i}\right)$ for $i<j$. As before, since $\partial$ is height decreasing, $\partial b_{j} \in \mathcal{A}_{Z}\left(a, b, a_{1}, \ldots, a_{r}, b_{1}, \ldots, b_{j-1}\right)$ and $\partial^{\prime} b_{j} \in \mathcal{A}_{Z}\left(a_{1}, \ldots, a_{r}, b_{1}, \ldots, b_{j-1}\right)$. By Lemma 2.16 we know that if $P$ is the sum of terms in $\partial b_{j}$ which do not contain $a$, then

$$
\partial b_{j}=P+\sum_{p} \epsilon\left(b_{j} ; Q_{p} a R_{p}\right) Q_{p} a R_{p}
$$

where $Q_{p}, R_{p} \in \mathcal{A}_{Z}\left(a_{1}, \ldots, a_{r}, b_{1}, \ldots, b_{j-1}\right)$. By the inductive assumption, $\Phi\left(b_{i}\right)$ does not contain $e_{1}$ for $i<j$ and so $\Phi\left(Q_{p}\right), \Phi\left(R_{p}\right)$, and $\Phi(P)$ do not contain $e_{1}$. So

$$
\begin{aligned}
H\left(\Phi\left(Q_{p} a R_{p}\right)\right) & =H\left(\Phi\left(Q_{p}\right)\left(-e_{1}+v\right) \Phi\left(R_{p}\right)\right) \\
& =(-1)^{\left|\Phi\left(Q_{p}\right)\right|} \Phi\left(Q_{p}\right) e_{2} \Phi\left(R_{p}\right) .
\end{aligned}
$$

Therefore

$$
H\left(\partial^{\prime} b_{j}-\Phi \partial b_{j}\right)=-\sum_{p}(-1)^{\left|\Phi\left(Q_{p}\right)\right|} \epsilon\left(b_{j} ; Q_{p} a R_{p}\right) \Phi\left(Q_{p}\right) e_{2} \Phi\left(R_{p}\right) .
$$

Thus $\Phi\left(b_{j}\right)=b_{j}+H\left(\partial^{\prime} b_{j}-\Phi \partial b_{j}\right)$ does not contain $e_{1}$.

We then see

$$
\begin{aligned}
\epsilon\left(b_{j}\right) & =\epsilon^{\prime} \Phi\left(b_{j}\right) \\
& =\epsilon^{\prime}\left(b_{j}+H\left(\partial^{\prime} b_{j}-\Phi \partial b_{j}\right)\right) \\
& =\epsilon^{\prime}\left(b_{j}\right)-\sum_{p}(-1)^{\left|\Phi\left(Q_{p}\right)\right|} \epsilon\left(b_{j} ; Q_{p} a R_{p}\right) \epsilon\left(Q_{p} b R_{p}\right),
\end{aligned}
$$

as desired. 
Therefore, after a type II move involved in making a dip, if $\epsilon\left(b_{i}\right)$ has been determined for $i<j$, then

$$
\epsilon\left(b_{j}\right)=\epsilon^{\prime}\left(b_{j}\right)-\sum(-1)^{\left|\Phi\left(Q_{p}\right)\right|} \epsilon\left(b_{j} ; Q_{p} a R_{p}\right) \epsilon\left(Q_{p} b R_{p}\right),
$$

where the sum is over totally augmented disks with positive corner at $b_{j}$ and a negative corner at $b$.

\section{Augmentation to Ruling}

In this section, we will use a construction similar to that of Sabloff's in [17] to construct a $\rho$-graded normal ruling from a $\rho$-graded augmentation to a fixed field $F$. This shows the forward direction of Theorem 1.1. Suppose that $D$ is the front diagram of a Legendrian knot $\Lambda$ in plat position. By the discussion in $\$ 2.5$ we can assume that there are base points $*_{1}, \ldots, *_{m}$, one on each right cusp, labeled from top to bottom corresponding to $t_{1}, \ldots, t_{m}$. Let $\epsilon^{\prime}: \mathcal{A}_{Z} \rightarrow F$ be a $\rho$-graded augmentation of the DGA $\left(\mathcal{A}_{Z}, \partial\right)$ over $Z=\mathbb{Z}\left[t_{1}^{ \pm 1}, \ldots, t_{m}^{ \pm 1}\right]$ of $D$. (Note that then $\epsilon^{\prime}(t)=\prod_{i=1}^{m} \epsilon^{\prime}\left(t_{i}\right)$ for the corresponding augmentation over $\mathbb{Z}\left[t, t^{-1}\right]$.) We will construct a $\rho$-graded normal ruling for the knot diagram while simultaneously extending the augmentation to an augmentation $\epsilon$ of the dipped diagram by adding one dip at a time from left to right. We will add base points to the diagram as we go to simplify the augmentation.

Start the ruling at the left of the diagram, pairing strands $2 k$ and $2 k-1$ for $1 \leqslant k \leqslant m$. We will extend the ruling from left to right along the diagram such that Property $(R)$, stated below, is satisfied. We can ensure Property (R) is satisfied because when introducing new crossings in the creation of the dips, the $a / b$-lattices, we get to choose where the augmentation sends the crossings in the $b$-lattice. We have enumerated the conditions we will need to check to ensure we end up with a $\rho$-graded augmentation of the dipped diagram and a $\rho$-graded normal ruling.

Property (R): At any dip, the generator $a_{r s}^{j}$ is augmented if and only if the strands $r$ and $s$ are paired in the ruling between $c_{j}$ and $c_{j+1}$.

Recall that the crossings from the resolution of the right cusps are labeled $q_{1}, \ldots, q_{m}$ from top to bottom and that the remaining crossings are labeled $c_{1}, \ldots, c_{n}$ from left to right. Also, the strands are labeled from bottom to top. It will also be important to recall that the orientation signs at positive original crossings are given by the left most diagram in Figure 5, while orientation signs at positive crossings in the $a / b$-lattices are given in the middle diagram.

We will inductively define augmentations on partially dipped diagrams by adding dips one at a time from left to right and defining augmentations on these diagrams. In particular, if $\epsilon_{j}$ is an augmentation on the diagram with dips added up to the $\operatorname{crossing} c_{j}$, we will extend the ruling and construct $\epsilon_{j+1}$, an augmentation on the diagram with dips added up to the crossing $c_{j+1}$ :

(1) Extend the ruling over $c_{j}$ by a switch if $\epsilon_{j}\left(c_{j}\right) \neq 0$ and just to the left of $c_{j}$, the ruling matches configuration (a), (b), or (c) in Figure 7 Otherwise, no switch.

(2) Consult Figure 12 to determine whether any base points will be added between $c_{j}$ and $c_{j+1}$. For each added base point, follow the strand it will end up on to the right all the way to a right cusp and add a base point $*_{\alpha}$ at the right cusp. Fix $\epsilon_{j+1}\left(t_{\alpha}\right)=-1$ and recall from $\$ 2.5$ that we must then set $\epsilon_{j+1}\left(t_{i}\right)=-\epsilon_{j}\left(t_{i}\right)$, where $*_{i}$ is the base point already at the right cusp $(1 \leqslant i \leqslant m)$. Move the base point $*_{\alpha}$ along the strand to between $c_{j}$ and $c_{j+1}$, modifying the augmentation on any crossing the base point goes over or under by a factor of -1 according to Remark 2.15 , 
(3) Place a dip between crossings $c_{j}$ and $c_{j+1}$, making sure to place the dip so that the new base points are to the right if they end up in the dip according to Figure 12 and to the left if not. Between each Reidemeister II move involved in making the dip:

(a) Extend the augmentation $\epsilon^{\prime}$ of the DGA of the diagram before the Reidemeister II move to an augmentation $\epsilon$ of the DGA of the new diagram satisfying Property (R) by defining $\epsilon$ on the two new crossings by Figure 12 and modifying $\epsilon$ from $\epsilon^{\prime}$ by Lemma 2.16 .

(b) Move base points to location specified by Figure 12 and modify $\epsilon$ using Remark 2.15 ,

Note that $\epsilon_{j+1}$ will agree with $\epsilon_{j}$ on the diagram to the left of $c_{j}$ though, according to Lemma 2.16, they may differ on $c_{j+1}, \ldots, c_{n}$.

When we complete this process and have a fully dipped diagram, the augmentation $\epsilon_{n}=\epsilon$ is a $\rho$-graded augmentation of the dipped diagram, and we have a normal ruling of the original diagram. We will also see that the resulting augmentation has restrictions on what $\epsilon(t)$ equals depending on whether $\rho$ is even or odd, yielding Theorem 3.1 and Theorem 1.2 .

For example, Figure 13 gives an augmentation to $\mathbb{R}$ of the right handed trefoil and the resulting ruling and augmentation of the dipped diagram from following this process.

3.1. Left cusps. Let $\epsilon_{0}$ be the $\rho$-graded augmentation of the original diagram. We know the ruling must pair strand $2 k$ with strand $2 k-1$ for $1 \leqslant k \leqslant m$ (where $m$ is the number of right cusps) at the left end of the diagram. Now add a dip between the left cusps and $c_{1}$. We must now extend $\epsilon_{0}$ to an augmentation $\epsilon_{1}$ of the new diagram. This will require successively extending the augmentation $\epsilon^{\prime}$ of the diagram before the Reidemeister II move to the augmentation $\epsilon$ of the diagram after one of the moves involved in constructing a dip. We will compute how the augmentation $\epsilon_{0}$ changes as we complete each Reidemeister II move in constructing the dip.

Consider the type II Reidemeister move which pushes strand $k$ over strand $\ell$. We must consider the following when extending $\epsilon^{\prime}$, the augmentation before pushing strand $k$ over strand $\ell$, to $\epsilon$, the augmentation of the resulting diagram.

(1) We must choose $\epsilon^{\prime}\left(e_{2}\right)$. In this case, choose $\epsilon^{\prime}\left(e_{2}\right)=0$. Thus, equation (2) tells us

$$
\epsilon\left(b_{k \ell}^{0}\right)=\epsilon^{\prime}\left(e_{2}\right)=0
$$

(2) By equation (2),

$$
\epsilon\left(a_{k \ell}^{0}\right)=\epsilon^{\prime}\left(v_{k \ell}\right)
$$

where

$$
\partial b_{k \ell}^{0}=a_{k \ell}^{0}+v_{k \ell} .
$$

From Figure 14 we know $v_{k \ell}$ is a sum of words in $b_{i j}^{0}$ for $(i, j)<(k, \ell)$ and contains a 1 if $(k, \ell)=$ $(2 r, 2 r-1)$ for some $1 \leqslant r \leqslant m$. Since $\epsilon^{\prime}\left(b_{i j}^{0}\right)=0$ for all $(i, j)<(k, \ell)$, by step $(1)$, we see that

$$
\epsilon\left(a_{k \ell}^{0}\right)=\epsilon^{\prime}\left(v_{k \ell}\right)= \begin{cases}1 & (k, \ell)=(2 r, 2 r-1) \text { for some } 1 \leqslant r \leqslant m \\ 0 & \text { otherwise. }\end{cases}
$$

(3) We must now check whether any "corrections" need to be made to $\epsilon^{\prime}$ to get $\epsilon$. In particular, whether there are any "corrections" which need to be made to $\epsilon^{\prime}$ on the $a_{i j}^{0}$ generators with $(i, j)<(k, \ell)$ but $i-j \geqslant k-\ell$. As $\epsilon^{\prime}\left(e_{2}\right)=0$, Lemma 2.16 tells us there are no corrections. 
$-(\mathrm{a})$

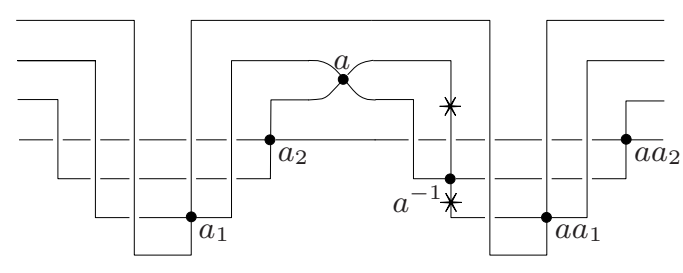

$-(\mathrm{b})$

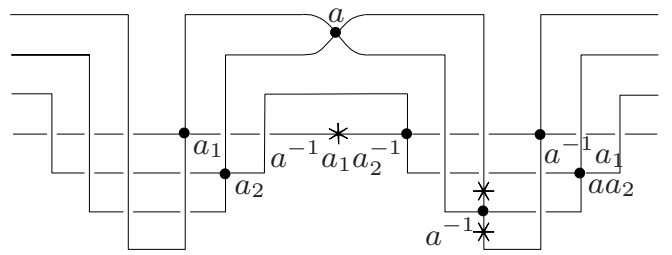

-(c), product of signs of $a_{L i}^{j-1}$ and $a_{i+1, K}^{j-1}$ is +1 $+(\mathrm{c})$, product of signs of $a_{L i}^{j-1}$ and $a_{i+1, K}^{j-1}$ is -1

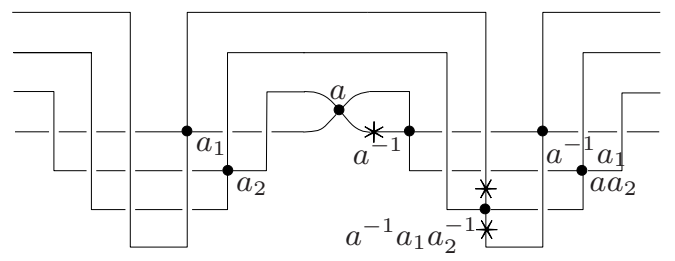

(d)

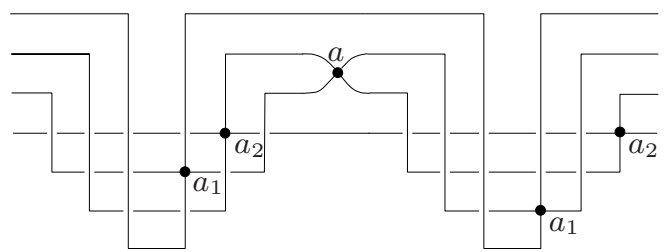

$-(\mathrm{e})$

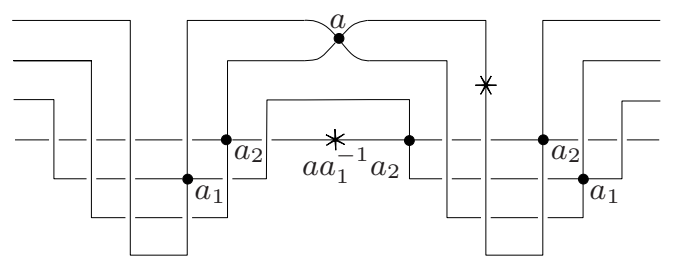

-(f), product of signs of $a_{L, i+1}^{j-1}$ and $a_{i K}^{j-1}$ is +1 $+(\mathrm{f})$, product of signs of $a_{L, i+1}^{j-1}$ and $a_{i K}^{j-1}$ is -1

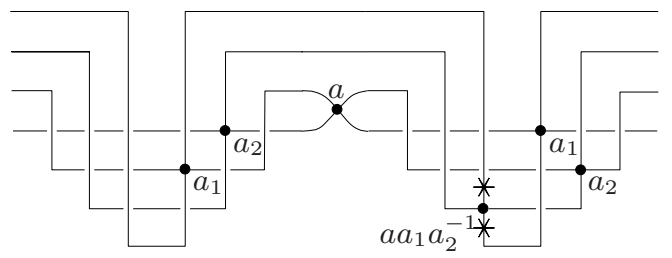

$+(\mathrm{a})$

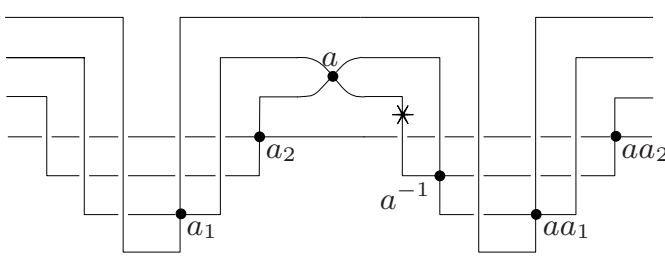

+ (b)

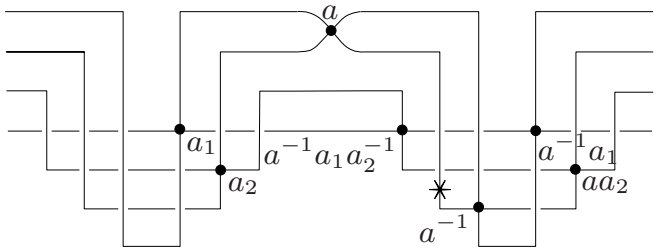

-(c), product of signs of $a_{L i}^{j-1}$ and $a_{i+1, K}^{j-1}$ is -1 $+(\mathrm{c})$, product of signs of $a_{L i}^{j-1}$ and $a_{i+1, K}^{j-1}$ is +1

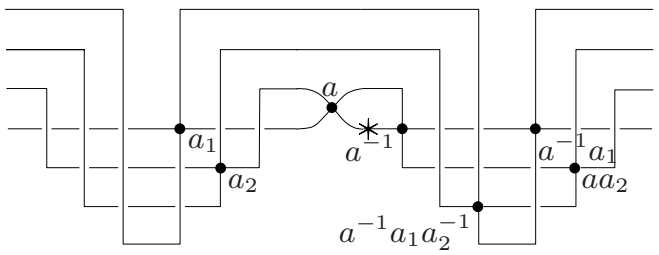

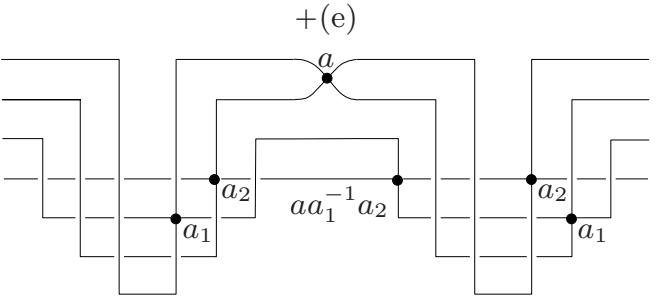

-(f), product of signs of $a_{L, i+1}^{j-1}$ and $a_{i K}^{j-1}$ is -1

$+(\mathrm{f})$, product of signs of $a_{L, i+1}^{j-1}$ and $a_{i K}^{j-1}$ is +1

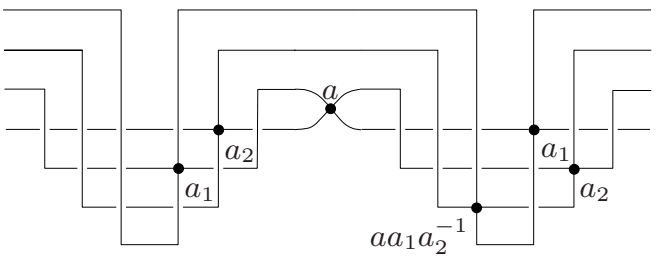

18

Figure 12. In the diagrams, * denotes a base point. A dot denotes the specified crossing is augmented and the augmentation sends the crossing to the label. Here $-/+(\mathrm{a})$ denotes a negative/positive crossing where the ruling has configuration (a) and the rest are defined analogously. 

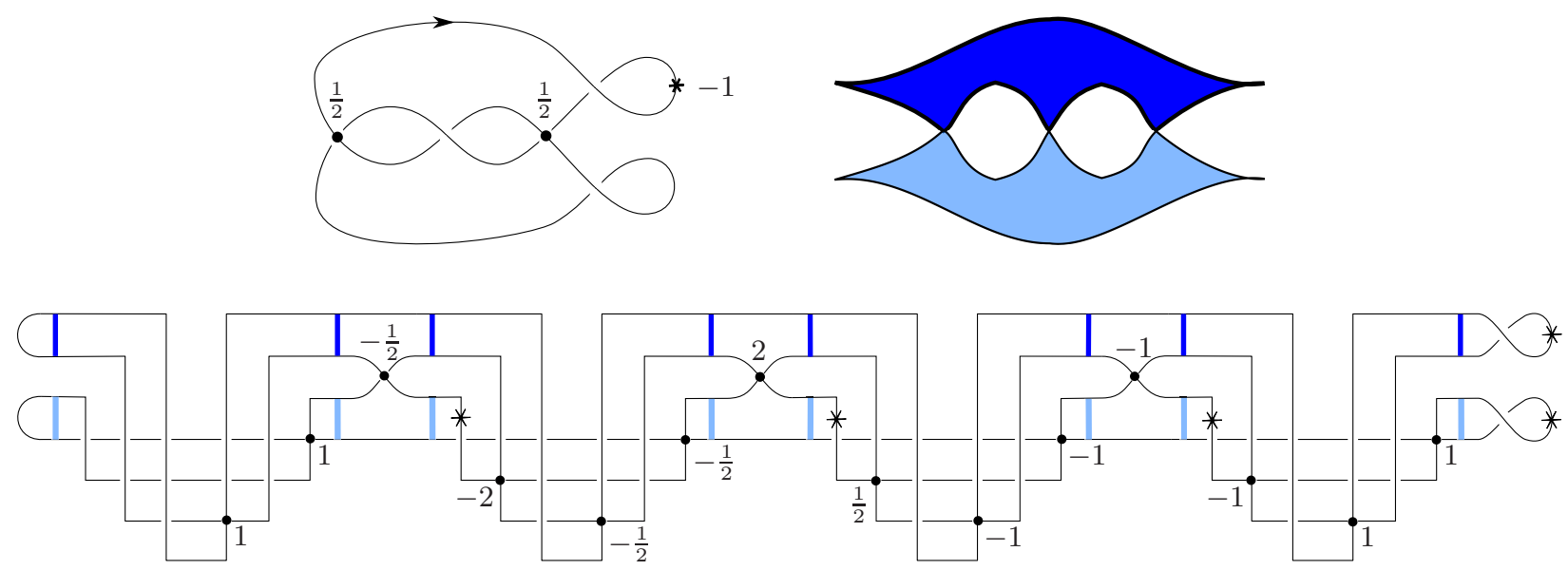

Figure 13. The top left diagram gives an augmentation of the right trefoil. The top right diagram gives the ruling and the bottom diagram gives the augmentation of the dipped diagram resulting from following the process of the proof. The dots denote that the crossing is augmented and the label on the dot gives where the augmentation sends the crossing. The * gives the placement of the base points. All base points are sent to -1 by the augmentation. (In general, it may not be the case that all base points are sent to -1 .)

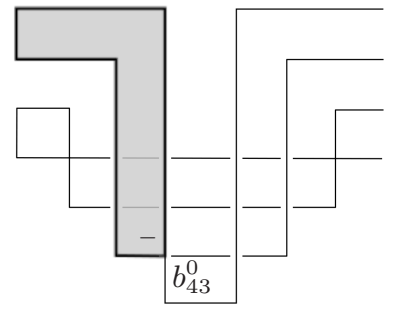

FiguRE 14. Shaded region gives the disk which contributes 1 to $\partial b_{43}^{0}$.

We must now check that the resulting augmentation is $\rho$-graded. We know

$$
\left|b_{2 r, 2 r-1}^{0}\right|=\mu(2 r)-\mu(2 r-1)=(\mu(2 r-1)+1)-\mu(2 r-1)=1
$$

for $1 \leqslant r \leqslant m$ and so

$$
\left|a_{2 r, 2 r-1}^{0}\right|=\left|b_{2 r, 2 r-1}^{0}\right|-1=0
$$

for $1 \leqslant r \leqslant m$. So if $\epsilon^{\prime}$ is $\rho$-graded, then $\epsilon$ is also and clearly $\epsilon$ is an augmentation satisfying Property (R).

3.2. Extending across original crossings. Consider the crossing $c_{j}$, the crossing of strands $i$ and $i+1$. Let us extend the ruling across the crossing $c_{j}$ and use $\epsilon_{j}$, the augmentation of the diagram with dips added up to the crossing $c_{j}$, to define $\epsilon_{j+1}$, the diagram with dips added up the crossing $c_{j+1}$. Note that $\epsilon_{j+1}$ will agree with $\epsilon_{j}$ on crossings to the left of the dip added between $c_{j}$ and $c_{j+1}$.

First we need to extend the ruling; extend the ruling across $c_{j}$ by a switch if $\epsilon_{j}\left(c_{j}\right) \neq 0$ and just to the left of $c_{j}$, the ruling so far matches configuration (a), (b), or (c). Otherwise, there is no switch. Let $1 \leqslant L, K \leqslant n$ such that strand $i$ is paired with strand $L$ and strand $i+1$ is paired with strand $K$ in the ruling between $c_{j}$ and $c_{j+1}$.

We will now construct a dip between between $c_{j}$ and $c_{j+1}$, move base points into place, and extend $\epsilon_{j}$ to an augmentation $\epsilon_{j+1}$ in the process. 
TABLE 1. All possible totally augmented disks.

\begin{tabular}{|c|c|c|}
\hline Configuration of $c_{j}$ & Positive corner & Terms in $\partial$ corresp. to totally aug. disks up to base pts. \\
\hline not augmented & $\begin{array}{l}b_{r s}^{j}, r, s \text { paired, } r, s \notin\{i, i+1\} \\
b_{\{i, L\}}^{j} \\
b_{\{i+1, K\}}^{j}\end{array}$ & $\begin{array}{l}a_{r s}^{j-1}, a_{r s}^{j} \\
a_{\{i+1, L\}}^{j-1}, a_{\{i, L\}}^{j} \\
a_{\{i, K\}}^{j-1}, a_{\{i+1, K\}}^{j}\end{array}$ \\
\hline (a) & $\begin{array}{l}b_{r s}^{j}, r, s \text { paired, } r, s \notin\{i, i+1\} \\
b_{i L}^{j} \\
b_{i+1, L}^{j} \\
b_{K i}^{j} \\
b_{K, i+1}^{j}\end{array}$ & $\begin{array}{l}a_{r s}^{j-1}, a_{r s}^{j} \\
c_{j} a_{i L}^{j-1}, a_{i L}^{j} \\
a_{i L}^{j-1}, b_{i+1, i}^{j} a_{i L}^{j} \\
a_{K, i+1}^{j-1}, a_{K, i+1}^{j-1} c_{j} b_{i+1, i}^{j} \\
a_{K, i+1}^{j} c_{j}, a_{K, i+1}^{j}\end{array}$ \\
\hline (b) & $\begin{array}{l}b_{r s}^{j}, r, s \text { paired, } r, s \notin\{i, i+1\} \\
b_{i K}^{j} \\
b_{i+1, K}^{j} \\
b_{i L}^{j} \\
b_{i+1, L}^{j}\end{array}$ & $\begin{array}{l}a_{r s}^{j-1}, a_{r s}^{j} \\
a_{i+1, K}^{j-1}, c_{j} a_{i L}^{j-1} b_{L K}^{j} \\
a_{i L}^{j-1} b_{L K}^{j}, a_{i+1, K}^{j} \\
c_{j} a_{i L}^{j-1}, a_{i L}^{j} \\
a_{i L}^{j-1}, b_{i+1, i}^{j} a_{i L}^{j}\end{array}$ \\
\hline (c) & $\begin{array}{l}b_{r s}^{j}, r, s \text { paired, } r, s \notin\{i, i+1\} \\
b_{K i}^{j} \\
b_{L i}^{j} \\
b_{K, i+1}^{j} \\
b_{L, i+1}^{j}\end{array}$ & $\begin{array}{l}a_{r s}^{j-1}, a_{r s}^{j} \\
a_{K, 1+1}^{j-1}, a_{K, i+1}^{j-1} c_{j} b_{i+1, i}^{j} \\
a_{L i}^{j-1} b_{i+1, i}^{j}, a_{L i}^{j} \\
a_{K, i+1}^{j-1} c_{j}, a_{K, i+1}^{j} \\
a_{L i}^{j-1}, b_{L K}^{j} a_{K, i+1}^{j}\end{array}$ \\
\hline (d) & $\begin{array}{l}b_{r s}^{j}, r, s \text { paired, } r, s \notin\{i, i+1\} \\
b_{i L}^{j} \\
b_{K, i+1}^{j}\end{array}$ & $\begin{array}{l}a_{r s}^{j-1}, a_{r s}^{j} \\
a_{i+1, L}^{j-1}, a_{i L}^{j} \\
a_{K i}^{j-1}, a_{K, i+1}^{j}\end{array}$ \\
\hline (e) & $\begin{array}{l}b_{r s}^{j}, r, s \text { paired, } r, s \notin\{i, i+1\} \\
b_{i L}^{j} \\
b_{i K}^{j} \\
b_{i+1, K}^{j}\end{array}$ & $\begin{array}{l}a_{r s}^{j-1}, a_{r s}^{j} \\
a_{i+1, L}^{j-1}, a_{i L}^{j} \\
c_{j} a_{i K}^{j-1}, a_{i+1, L}^{j-1} b_{L K}^{j} \\
a_{i K}^{j-1}, a_{i+1, K}^{j}\end{array}$ \\
\hline$(\mathrm{f})$ & $\begin{array}{l}b_{r r}^{j}, r, s \text { paired, } r, s \notin\{i, i+1\} \\
b_{L i}^{j} \\
b_{K, i+1}^{j} \\
b_{L, i+1}^{j}\end{array}$ & $\begin{array}{l}a_{r s}^{j-1}, a_{r s}^{j} \\
a_{L, i+1}^{j-1}, a_{L i}^{j} \\
a_{K i}^{j-1}, a_{K, i+1}^{j} \\
a_{L i}^{j-1} c_{j}, b_{L K}^{j} a_{K, i+1}^{j}\end{array}$ \\
\hline
\end{tabular}

It will be useful to note that Table1gives all possibly totally augmented disks in the various configurations of the ruling near crossings, up to base points. 


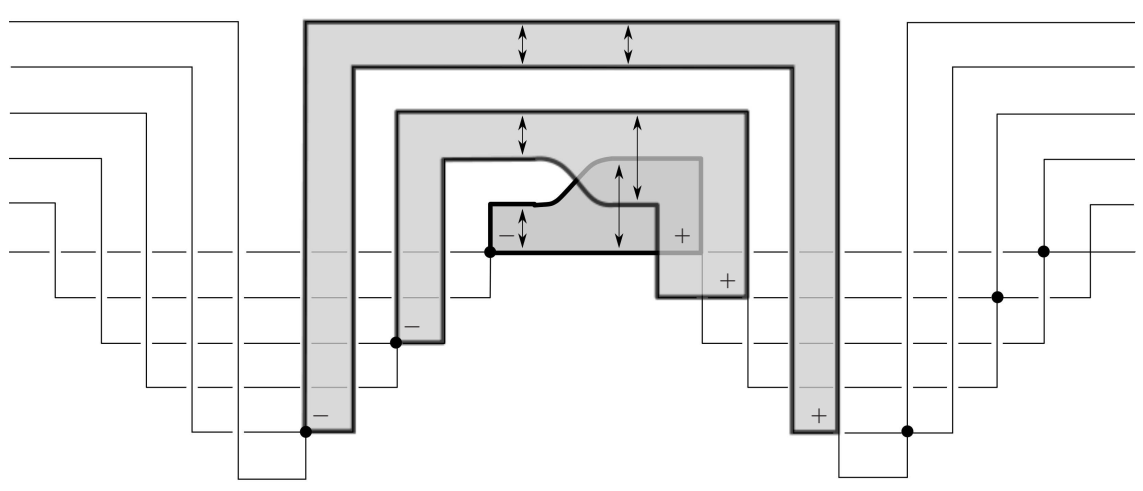

FiguRE 15. The disks with one negative corner in the $a^{k-1}$-lattice which contribute terms to the differential of crossings in the $b^{k}$-lattice if $\epsilon_{j}\left(c_{j}\right)=0$.

Since the way we extend the ruling across $c_{j}$ depends on $\epsilon_{j}\left(c_{j}\right)$ and the ruling immediately to the left of $c_{j}$, we will need to consider when $\epsilon_{j}\left(c_{j}\right)=0$ and $\epsilon_{j}\left(c_{j}\right) \neq 0$.

(Case 1: $\left.\epsilon_{j}\left(c_{j}\right)=0\right)$ In this case, extend the ruling across $c_{j}$ without a switch. As with adding a dip between the left cusps and $c_{1}$, we will compute how the augmentation $\epsilon^{\prime}$ of the diagram before a Reidemeister II move changes to an augmentation $\epsilon$ after each move involved in the making the dip. Consider the type II move that pushes strand $k$ over strand $\ell$. Let $\epsilon^{\prime}$ be the augmentation on the diagram before the move and let $\epsilon$ be the augmentation on the resulting diagram. We will proceed as follows:

(1) Define $\epsilon$ on the $b^{j}$-lattice.

(2) Define $\epsilon$ on the $a^{j}$-lattice.

(3) Make corrections to $\epsilon$ using Lemma 2.16.

(4) Make corrections due to moving base points into place.

Following this process, we have:

(1) Choose $\epsilon^{\prime}\left(e_{2}\right)=0$.

(2) From equation (2), we know

$$
\epsilon\left(a_{k \ell}^{j}\right)=\epsilon^{\prime}\left(v_{k \ell}\right) \text {. }
$$

Since neither $c_{j}$ nor any crossing in the $b^{j}$-lattice is augmented, the only totally augmented disks in $v_{k \ell}$ have a positive corner at $b_{k \ell}^{j}$ and a single augmented negative corner in the $a^{j-1}$-lattice.

If such a disk exists, by Property (R), the negative corner in the $a^{j-1}$-lattice must be where two paired strands in the ruling cross as seen in Figure 15. Since this is the only negative corner of the disk, we know $k$ and $\ell$ are paired in the ruling between $c_{j}$ and $c_{j+1}$ as well. So, if we recall that $a_{\{k, \ell\}}^{j}=a_{\max (k, \ell), \min (k, \ell)}^{j}$, then

$$
\begin{aligned}
\epsilon\left(a_{k \ell}^{j}\right)= & \epsilon^{\prime}\left(v_{k \ell}\right)=\epsilon^{\prime}\left(\epsilon\left(b_{k \ell}^{j} ; a_{k \ell}^{j-1}\right) a_{k \ell}^{j-1}\right) \\
= & \begin{cases}\epsilon\left(a_{\{i, K\}}^{j-1}\right) & (k, \ell)=\{i+1, K\} \\
\epsilon\left(a_{\{i-1, L\}}^{j-1}\right) & (k, \ell)=\{i, L\} \\
\epsilon\left(a_{k \ell}^{j-1}\right) & \text { if } k, \ell \text { paired and } k, \ell \neq i, i+1 \\
0 & \text { otherwise. }\end{cases}
\end{aligned}
$$

(3) Since $\epsilon^{\prime}\left(e_{2}\right)=0$, by Lemma 2.16, we know there are no "corrections" to $\epsilon\left(a_{r s}^{j}\right)$ for $(r, s)<(k, \ell)$.

(4) As there are no base points to move into place, no modifications to the augmentation are needed. 
We must now check that the resulting augmentation is $\rho$-graded. Since $\epsilon^{\prime}$ satisfies Property (R), we know $a_{\{i, K\}}^{j-1}, a_{\{i+1, L\}}^{j-1}$, and $a_{k \ell}^{j-1}$ are augmented if strands $k$ and $\ell$ are paired between $c_{j}$ and $c_{j+1}$. Thus, if $\epsilon^{\prime}$ is a $\rho$-graded augmentation, then each has degree divisible by $\rho$. Since $\partial$ lowers degree by one,

$$
\left|b_{\{i+1, K\}}^{j}\right|=\left|a_{\{i, K\}}^{j-1}\right|+1, \quad\left|b_{\{i, L\}}^{j}\right|=\left|a_{\{i+1, L\}}^{j-1}\right|+1, \quad\left|b_{k \ell}^{j}\right|=\left|a_{k \ell}^{j-1}\right|+1
$$

and since $\left|a_{r s}^{j}\right|=\left|b_{r s}^{j}\right|-1$,

$$
\left|a_{\{i+1, K\}}^{j}\right|=\left|a_{\{i, K\}}^{j-1}\right|, \quad\left|a_{\{i, L\}}^{j}\right|=\left|a_{\{i+1, L\}}^{j-1}\right|, \quad\left|a_{k \ell}^{j}\right|=\left|a_{k \ell}^{j-1}\right| .
$$

So $\epsilon$ is a $\rho$-graded augmentation satisfying Property (R) if $\epsilon^{\prime}$ is $\rho$-graded.

(Case 2: $\left.\epsilon_{j}\left(c_{j}\right) \neq 0\right)$ Now suppose $c_{j}$ is augmented. This breaks into six cases, one for each possible configuration of $c_{j}$ seen in Figure 7 In each case, while creating the dip, we will extend the augmentation $\epsilon_{j}$ of the knot diagram before adding the dip between crossings $c_{j}$ and $c_{j+1}$ over the dip, move the base points into place and modify the augmentation accordingly to end up with an augmentation $\epsilon_{j+1}$ of the modified diagram. As in the case where $c_{j}$ was not augmented, we will compute how the augmentation changes as we do each Reidemeister II move involved in making a dip between $c_{j}$ and $c_{j+1}$.

Configuration (a): By considering Figure 12 we see that if $c_{j}$ is a negative crossing, we add two base points at the right cusp to the right on strand $i+1$ and move them along strand $i+1$ to between $c_{j}$ and $c_{j+1}$, modifying the augmentation on any crossings we push the base points over/under according to Remark 2.15 Note that as we are moving two base points along the same strand, no modification of the augmentation is necessary. If $c_{j}$ is a positive crossing, we add one base point on strand $i$ and follow the same process, though, in this case, modification of the augmentation by a factor of -1 on the crossings we push the base point over/under is necessary by Remark 2.15. Note that whether $c_{j}$ is a positive or negative crossing, one base point will be to the left of the dip we are adding, and, if $c_{j}$ is a negative crossing, we will also have one base point to the right.

Consider the Reidemeister II move where strand $k$ is pushed over strand $\ell$. Let $\epsilon^{\prime}$ be the augmentation on the diagram before the move and let $\epsilon$ be the augmentation of the diagram after. Note that by our strand labeling convention $L<i<i+1<K$.

As before, we must consider the following:

$(k, \ell)<(i+1, i):$

(1) Choose $\epsilon^{\prime}\left(e_{2}\right)=0$.

(2) We know $\epsilon\left(a_{k \ell}^{j}\right)=\epsilon^{\prime}\left(v_{k \ell}\right)$. If $k \neq i, i+1$, then Table 1 tells us

$$
\epsilon^{\prime}\left(v_{k \ell}\right)=\epsilon^{\prime}\left(\epsilon\left(b_{k \ell}^{j} ; a_{k \ell}^{j-1}\right) a_{k \ell}^{j-1}\right) .
$$

So, in this case, $v_{k \ell}$ has a totally augmented disk if and only if $\epsilon^{\prime}\left(a_{k \ell}^{j-1}\right) \neq 0$ if and only if $k$ and $\ell$ are paired between $c_{j-1}$ and $c_{j+1}$ by Property $(\mathrm{R})$. Otherwise $(k, \ell)=(i+1, L)$ or $(k, \ell)=(i, L)$. In these cases

$$
\begin{aligned}
& \epsilon\left(a_{i L}^{j}\right)=\epsilon^{\prime}\left(v_{i L}\right) \\
& = \begin{cases}\epsilon^{\prime}\left(\epsilon\left(b_{i L}^{j} ; c_{j} a_{i L}^{j-1}\right) c_{j} a_{i L}^{j-1}\right) & c_{j} \text { negative crossing } \\
\epsilon^{\prime}\left(\epsilon\left(b_{i L}^{j} ; c_{j} a_{i L}^{j-1}\right) t_{\alpha}^{ \pm 1} c_{j} a_{i L}^{j-1}\right) & c_{j} \text { positive crossing }\end{cases} \\
& =\epsilon\left(c_{j} a_{i L}^{j-1}\right)
\end{aligned}
$$


and

$$
\begin{aligned}
\epsilon\left(a_{i+1, L}^{j}\right) & =\epsilon^{\prime}\left(v_{i+1, L}\right) \\
& = \begin{cases}\epsilon^{\prime}\left(\epsilon\left(b_{i+1, L}^{j} ; a_{i L}^{j-1}\right) t_{\alpha}^{ \pm 1} a_{i L}^{j-1}\right) & c_{j} \text { negative crossing } \\
\epsilon^{\prime}\left(\epsilon\left(b_{i+1, L}^{j} ; a_{i L}^{j-1}\right) a_{i L}^{j-1}\right) & c_{j} \text { positive crossing }\end{cases} \\
& = \begin{cases}-\epsilon\left(a_{i L}^{j-1}\right) & c_{j} \text { negative crossing } \\
\epsilon\left(a_{i L}^{j-1}\right) & c_{j} \text { positive crossing. }\end{cases}
\end{aligned}
$$

(3) Since $\epsilon^{\prime}\left(e_{2}\right)=0$ by Lemma 2.16, there are no "corrections" to the augmentation of the previously constructed portion of the $a^{j}$-lattice.

(4) In the case where $c_{j}$ is a negative crossing, according to Figure 12 we move a base point over $a_{i+1, L}^{j}$ to get

$$
\epsilon\left(a_{i+1, L}^{j}\right)=\left\{\begin{array}{ll}
-\left(-\epsilon\left(a_{i L}^{j-1}\right)\right) & c_{j} \text { negative crossing } \\
\epsilon\left(a_{i L}^{j-1}\right) & c_{j} \text { positive crossing }
\end{array}\right\}=\epsilon\left(a_{i L}^{j-1}\right) .
$$

Note that we do not need to move the other base points as they are to the left of the dip and so no more modifications are necessary.

$(k, \ell)=(i+1, i):$

(1) According to Figure 12, choose $\epsilon^{\prime}\left(e_{2}\right)=\left(\epsilon\left(c_{j}\right)\right)^{-1}$. Then $\epsilon\left(b_{i+1, i}^{j}\right)=\epsilon^{\prime}\left(e_{2}\right)=\left(\epsilon\left(c_{j}\right)\right)^{-1}$.

(2) From looking at Table 1, we see that $v_{i+1, i}=0$ and so $\epsilon\left(a_{i+1, i}^{j}\right)=\epsilon^{\prime}\left(v_{i+1, i}\right)=0$.

(3) As $\epsilon^{\prime}\left(e_{2}\right)=1$ we need to check for "corrections." In particular, the disk in Figure 16 contributes the term $a_{i+1, i}^{j} a_{i L}^{j}$ to $\partial a_{i+1, L}^{j}$ and is the only disk with negative corner at $a_{i+1, i}^{j}$ whose other negative corners are augmented since $a_{i L}^{j}$ is the only crossing of strand $L$ which is augmented by Property (R). Thus Lemma 2.16 tells us

$$
\begin{aligned}
& \epsilon\left(a_{i+1, L}^{j}\right)= \begin{cases}\epsilon^{\prime}\left(a_{i+1, L}^{j}\right)-(-1)^{\left|t_{\alpha}^{ \pm 1}\right|} \epsilon\left(a_{i+i, L}^{j} ; a_{i+1, i}^{j} a_{i L}^{j}\right) \epsilon\left(t_{\alpha}^{ \pm 1} b_{i+1, i}^{j} a_{i L}^{j}\right) & c_{j} \text { negative crossing } \\
\epsilon^{\prime}\left(a_{i+1, L}^{j}\right)-(-1)^{|1|} \epsilon\left(a_{i+i, L}^{j} ; a_{i+1, i}^{j} a_{i L}^{j}\right) \epsilon\left(b_{i+1, i}^{j} a_{i L}^{j}\right) & c_{j} \text { positive crossing }\end{cases} \\
& = \begin{cases}\epsilon\left(a_{i L}^{j-1}\right)+\epsilon\left(t_{\alpha}^{ \pm 1} b_{i+1, i}^{j} a_{i L}^{j}\right) & c_{j} \text { negative crossing } \\
\epsilon\left(a_{i L}^{j-1}\right)+\epsilon\left(b_{i+1, i}^{j} a_{i L}^{j}\right) & c_{j} \text { positive crossing }\end{cases} \\
& =\epsilon\left(a_{i L}^{j-1}\right)-\left(\epsilon\left(c_{j}\right)\right)^{-1} \epsilon\left(c_{j} a_{i L}^{j-1}\right) \\
& =0 \text {, }
\end{aligned}
$$

where $t_{\alpha}$ is associated with the base point $*$, since

$$
\epsilon\left(a_{i+1, L}^{j} ; a_{i+1, i}^{j} a_{i L}^{j}\right)= \begin{cases}-1 & c_{j} \text { negative crossing } \\ 1 & c_{j} \text { positive crossing. }\end{cases}
$$

Thus $\epsilon$ satisfies Property (R).

(4) By Remark 2.15, moving a base point over $a_{i+1, i}^{j}$ will not change the augmentation since $\epsilon\left(a_{i+1, i}^{j}\right)=0$ in the case where $c_{j}$ is a negative crossing.

$(k, \ell)>(i+1, i):$

(1) According to Figure 12, choose $\epsilon^{\prime}\left(e_{2}\right)=0$. 


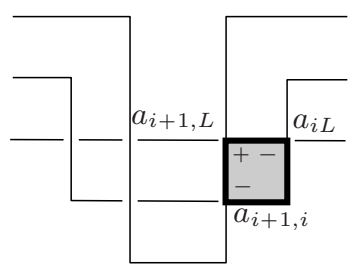

Figure 16. The disk contributing to $\partial a_{i+1, L}^{j}$, which requires "correcting" the augmentation. Crossings are labeled.
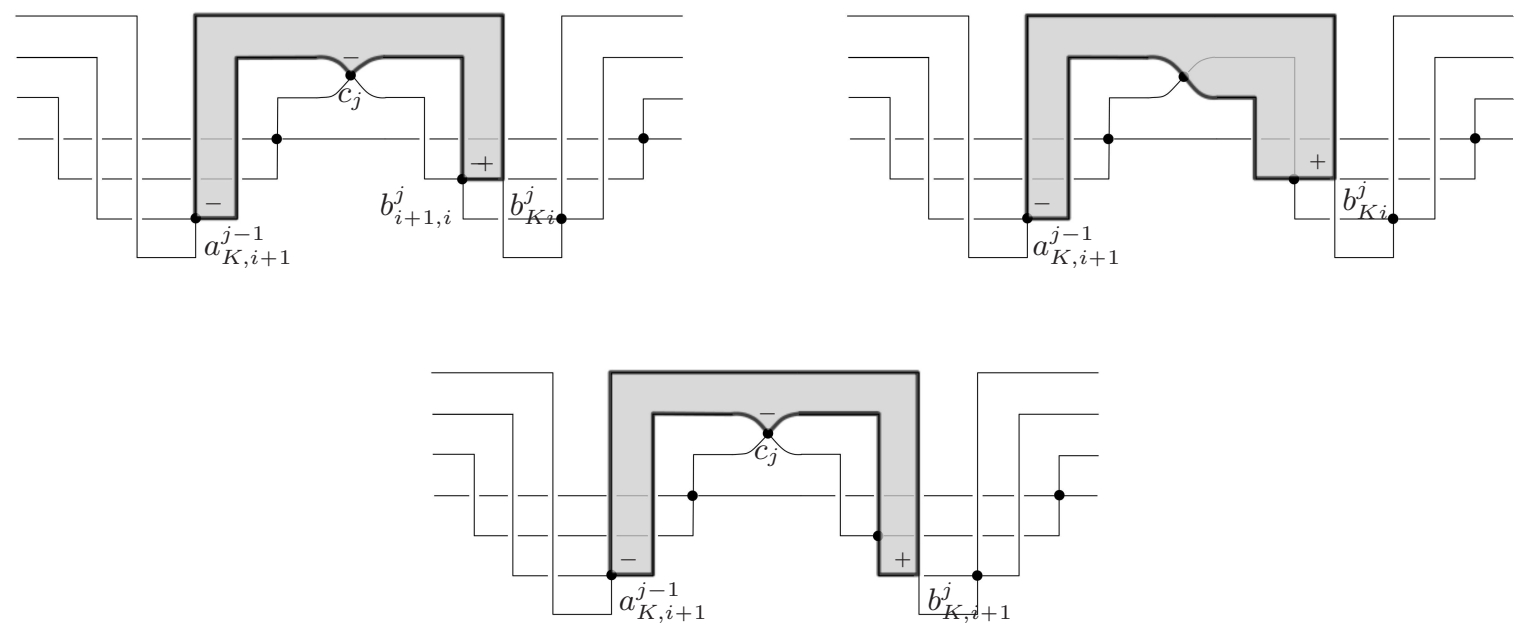

FiguRE 17. Totally augmented disks with one negative corner in the $a^{j-1}$-lattice contributing to the differential of crossings in the $b^{j}$-lattice. The crossings at corners of the disks are labeled.

(2) As before, if neither strands $k$ nor $\ell$ is a crossing strand, then $a_{k \ell}^{j}$ is augmented if and only if $k$ and $\ell$ are paired in the ruling between $c_{j}$ and $c_{j+1}$. Note that this tells us the augmentation on the $a^{j}$-lattice is the same as the $a^{j-1}$-lattice. We do, however, see in Figure 17 that there is one totally augmented disk in $v_{K, i+1}$ and two in $v_{K i}$.

Thus

$$
\begin{aligned}
& \epsilon\left(a_{K i}^{j}\right)=\epsilon^{\prime}\left(v_{K i}\right) \\
& = \begin{cases}\epsilon^{\prime}\left(\epsilon\left(b_{K i}^{j} ; a_{K, i+1}^{j-1}\right) a_{K, i+1}^{j-1}\right)+\epsilon^{\prime}\left(\epsilon\left(b_{K i}^{j} ; a_{K, i+1}^{j-1} c_{j} b_{i+1, i}^{j}\right) a_{K, i+1}^{j-1} c_{j} t_{\alpha}^{ \pm 1} b_{i+1, i}^{j}\right) & c_{j} \text { negative crossing } \\
\epsilon^{\prime}\left(\epsilon\left(b_{K i}^{j} ; a_{K, i+1}^{j-1}\right) a_{K, i+1}^{j-1} t_{\alpha}^{ \pm 1}\right)+\epsilon^{\prime}\left(\epsilon\left(b_{K i}^{j} ; a_{K, i+1}^{j-1} c_{j} b_{i+1, i}^{j}\right) a_{K, i+1}^{j-1} c_{j} b_{i+1, i}^{j}\right) & c_{j} \text { positive crossing }\end{cases} \\
& = \begin{cases}\epsilon\left(a_{K, i+1}^{j-1}\right)-\epsilon\left(a_{K, i+1}^{j-1} c_{j} b_{i+1, i}^{j}\right) & c_{j} \text { negative crossing } \\
-\epsilon\left(a_{K, i+1}^{j-1}\right)+\epsilon\left(a_{K, i+1}^{j-1} c_{j} b_{i+1, i}^{j}\right) & c_{j} \text { positive crossing }\end{cases} \\
& =0 \text {, }
\end{aligned}
$$

since

$$
\epsilon\left(a_{K, i+1}^{j} c_{j} b_{i+1, i}^{j}\right)=\epsilon\left(a_{K, i+1}^{j-1} c_{j}\right)\left(\epsilon\left(c_{j}\right)\right)^{-1}=\epsilon\left(a_{K, i+1}^{j-1}\right) .
$$


And,

$$
\begin{aligned}
\epsilon\left(a_{K, i+1}^{j}\right) & =\epsilon^{\prime}\left(v_{K, i+1}\right) \\
& = \begin{cases}\epsilon^{\prime}\left(\epsilon\left(b_{K, i+1}^{j} ; a_{K, i+1}^{j-1} c_{j}\right) a_{K, i+1}^{j-1} c_{j} t_{\alpha}^{ \pm 1} t_{\beta}^{ \pm 1}\right) & c_{j} \text { negative crossing } \\
\epsilon^{\prime}\left(\epsilon\left(b_{K, i+1}^{j} ; a_{K, i+1}^{j-1} c_{j}\right) a_{K, i+1}^{j-1} c_{j}\right) & c_{j} \text { positive crossing }\end{cases} \\
& =\epsilon\left(a_{K, i+1}^{j-1} c_{j}\right) .
\end{aligned}
$$

(3) Since $\epsilon^{\prime}\left(e_{2}\right)=0$, by Lemma 2.16, no "corrections."

(4) By Figure 12, no base points to move.

If $\epsilon^{\prime}$ is a $\rho$-graded augmentation, then $\rho|| c_{j} \mid$ since $c_{j}$ is augmented. Thus, the ruling is $\rho$-graded so far. We see that $\left|b_{i+1, i}^{j}\right|=\mu(i+1)-\mu(i)=\left|c_{j}\right|$ and, since $\partial$ lowers degree by one,

$$
\begin{aligned}
& \left|a_{K, i+1}^{j}\right|=\left|b_{K, i+1}^{j}\right|-1=\left|a_{K, i+1}^{j-1}\right| \\
& \left|a_{i L}^{j}\right|=\left|b_{i L}^{j}\right|-1=\left|a_{i L}^{j-1}\right| .
\end{aligned}
$$

As in the nonaugmentated case, if strands $k$ and $\ell$ are paired in the ruling between $c_{j-1}$ and $c_{j+1}$, then $a_{k \ell}^{j-1}$ is augmented and $\left|a_{k \ell}^{j}\right|=\left|a_{k \ell}^{j-1}\right|$. So $\epsilon$ is a $\rho$-graded augmentation which satisfies Property (R).

Configuration (b): Now suppose the ruling has configuration (b) near $c_{j}$. Note that with our strand assignments $i+1>i>L>K$. According to Figure 12, if $c_{j}$ is a negative crossing, then follow strand $K$ to the right to a right cusp and add a base point and follow strand $i+1$ to the right to a right cusp and add two base points. Move these base points back along their respective strands to between $c_{j}$ and $c_{j+1}$, modifying the augmentation according to Remark 2.15. If $c_{j}$ is a positive crossing, then follow strand $i$ to the right to a right cusp, add a base point, and move it back to between $c_{j}$ and $c_{j+1}$, modifying the augmentation as necessary.

As before, we will compute how the augmentation $\epsilon_{j}$ changes as we complete Reidemeister II moves involved in the construction of a dip, to yield the extended augmentation $\epsilon_{j+1}$.

Consider the augmentation $\epsilon$ extension of the augmentation $\epsilon^{\prime}$ where strand $k$ is pushed over strand $\ell$ in the creation of a dip between $c_{j}$ and $c_{j+1}$.

$(k, \ell)<(L, K)$ : This case follows in the way of the first case of configuration (a) so that setting $\epsilon^{\prime}\left(e_{2}\right)=0$, we transfer the augmentation on the $a^{j-1}$-lattice to that $a^{j}$-lattice.

$(k, \ell)=(L, K):$

(1) According to Figure 12, set $\epsilon^{\prime}\left(e_{2}\right)=\left(\epsilon\left(c_{j} a_{i L}^{j-1}\right)\right)^{-1} \epsilon\left(a_{i+1, K}^{j-1}\right)$ to obtain $\epsilon\left(b_{L K}^{j}\right)=\epsilon^{\prime}\left(e_{2}\right)=\left(\epsilon\left(c_{j} a_{i L}^{j-1}\right)\right)^{-1} \epsilon\left(a_{i+1, K}^{j-1}\right)$.

(2) We see that $\epsilon^{\prime}\left(v_{L K}\right)=0$, since $K$ and $L$ are neither paired nor crossing strands in the ruling between $c_{j}$ and $c_{j+1}$. Thus

$$
\epsilon\left(a_{L K}^{j}\right)=\epsilon^{\prime}\left(v_{L K}\right)=0
$$

(3) There are no "corrections" as any disk in the $a_{j}$-lattice with negative corner at $a_{L K}^{j}$ must have an augmented negative corner of the form $a_{L *}^{j}$, but strand $L$ is paired with strand $i$ in the ruling between $c_{j}$ and $c_{j+1}$, so the only such crossing has not been made in the dip yet.

(4) No base points to move, so no corrections.

$(L, K)<(k, \ell)<(i+1, i):$

(1) According to Figure 12, set $\epsilon^{\prime}\left(e_{2}\right)=0$. 

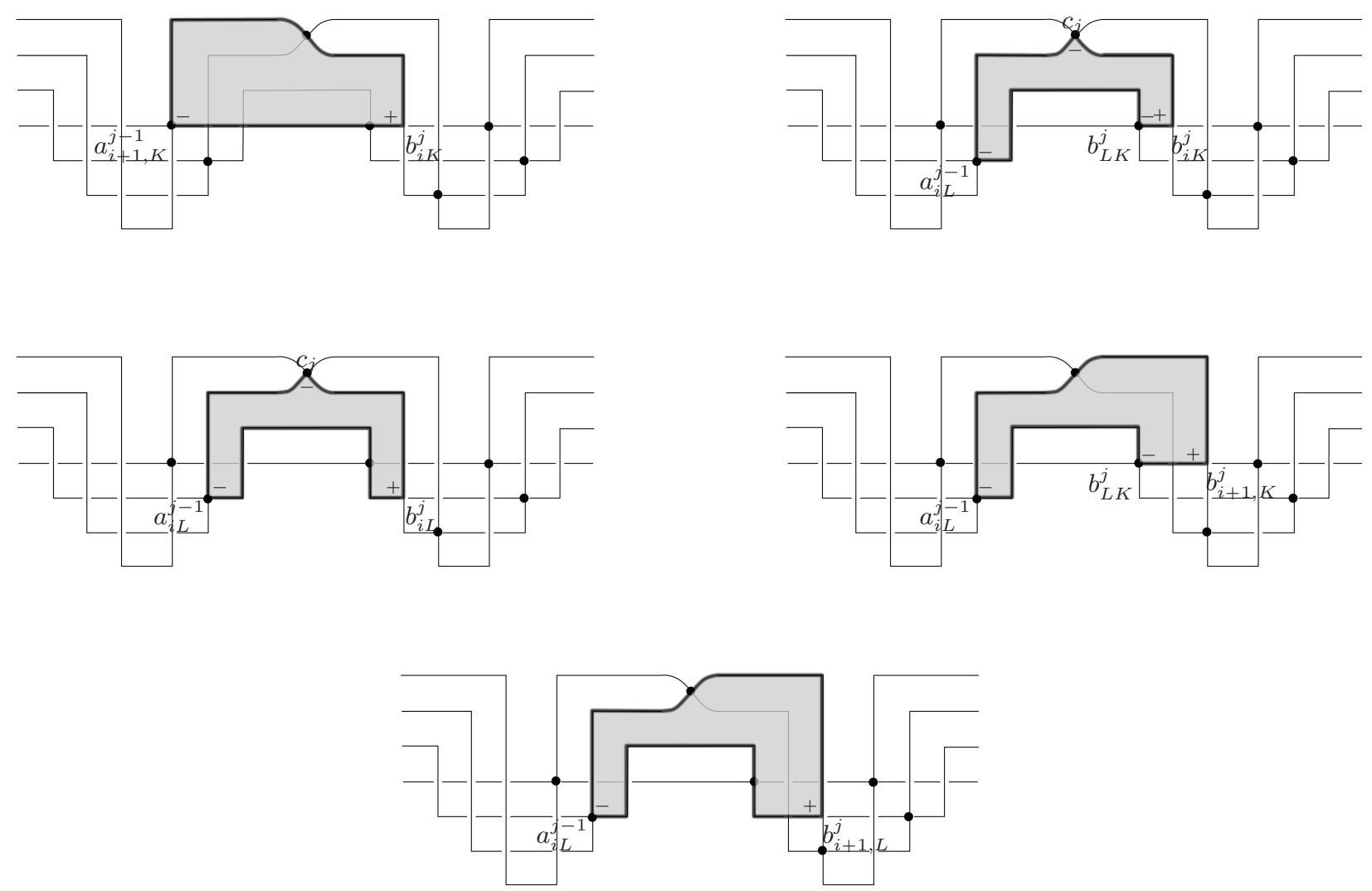

FIGURE 18. Totally augmented disks with one negative corner in the $b^{j-1}$-lattice which contribute to the differential of a crossing in the $a^{j}$-lattice. All crossings at corners of disks are labeled.

(2) In Figure 18, we see all the totally augmented disks contributing to $v_{k \ell}$ in $\partial b_{k \ell}^{j}$.

Therefore

$$
\begin{aligned}
& \epsilon\left(a_{i K}^{j}\right)=\epsilon^{\prime}\left(v_{i K}\right) \\
& = \begin{cases}\epsilon^{\prime}\left(\epsilon\left(b_{i K}^{j} ; a_{i+1, K}^{j-1}\right) a_{i+1, K}^{j-1} t_{\alpha}^{ \pm 1}\right)+\epsilon^{\prime}\left(\epsilon\left(b_{i K}^{j} ; c_{j} a_{i L}^{j-1} b_{L K}^{j}\right) c_{j} a_{i L}^{j-1} b_{L K}^{j}\right) & c_{j} \text { negative crossing } \\
\epsilon^{\prime}\left(\epsilon\left(b_{i K}^{j} ; a_{i+1, K}^{j-1}\right) a_{i+1, K}^{j-1}\right)+\epsilon^{\prime}\left(\epsilon\left(b_{i K}^{j} ; c_{j} a_{i L}^{j-1} b_{L K}^{j}\right) c_{j} a_{i L}^{j-1} b_{L K}^{j}\right) & c_{j} \text { positive crossing }\end{cases} \\
& = \begin{cases}-\epsilon\left(a_{i+1, K}^{j-1}\right)+\epsilon\left(c_{j} a_{i L}^{j-1} b_{L K}^{j}\right) & c_{j} \text { negative crossing } \\
-\epsilon\left(a_{i+1, K}^{j-1}\right)-\epsilon\left(c_{j} a_{i L}^{j-1} b_{L K}^{j}\right) & c_{j} \text { positive crossing }\end{cases} \\
& =0 \text {, }
\end{aligned}
$$

since

$$
\epsilon\left(c_{j} a_{i L}^{j-1} b_{L K}^{j}\right)=\epsilon\left(c_{j} a_{i L}^{j-1}\right)\left(\epsilon\left(c_{j} a_{i L}^{j-1}\right)\right)^{-1} \epsilon\left(a_{i+1, K}^{j-1}\right)=\epsilon\left(a_{i+1, K}^{j-1}\right) .
$$


We also have

$$
\begin{aligned}
\epsilon\left(a_{i L}^{j}\right) & =\epsilon^{\prime}\left(v_{i K}\right)=\epsilon^{\prime}\left(\epsilon\left(b_{i L}^{j} ; c_{j} a_{i L}^{j-1}\right) c_{j} a_{i L}^{j-1}\right) \\
& = \begin{cases}\epsilon\left(c_{j} a_{i L}^{j-1}\right) & c_{j} \text { negative crossing } \\
-\epsilon\left(c_{j} a_{i L}^{j-1}\right) & c_{j} \text { positive crossing, }\end{cases} \\
\epsilon\left(a_{i+1, K}^{j}\right) & =\epsilon^{\prime}\left(v_{i+1, K}\right)=\epsilon^{\prime}\left(\epsilon\left(b_{i+1, K}^{j} ; a_{i L}^{j-1} b_{L K}^{j}\right) c_{j} a_{i L}^{j-1}\right) \\
& =\epsilon\left(a_{i L}^{j-1} b_{L K}^{j}\right) \\
& =\epsilon\left(a_{i L}^{j-1}\right)\left(\epsilon\left(c_{j} a_{i L}^{j-1}\right)\right)^{-1} \epsilon\left(a_{i+1, K}^{j}\right), \\
\epsilon\left(a_{i+1, L}^{j}\right) & =\epsilon^{\prime}\left(v_{i+1, L}\right)=\epsilon^{\prime}\left(\epsilon\left(b_{i+1, L}^{j} ; a_{i L}^{j-1}\right) a_{i L}^{j-1}\right)=\epsilon\left(a_{i L}^{j-1}\right) .
\end{aligned}
$$

(3) Since $\epsilon^{\prime}\left(e_{2}\right)=0$, by Lemma 2.16, there are no "corrections."

(4) Note that if $c_{j}$ is a negative crossing, according to Figure 12, we need to move two base points over $a_{i+1, K}^{j}$ and $a_{i+1, L}^{j}$, so no changes. However, if $c_{j}$ is a positive crossing, then we need to move one base point over $a_{i K}^{j}$ and $a_{i L}^{j}$ to give $\epsilon\left(a_{i K}^{j}\right)=0$ and

$$
\epsilon\left(a_{i L}^{j}\right)=\left\{\begin{array}{ll}
\epsilon\left(c_{j} a_{i L}^{j-1}\right) & c_{j} \text { negative crossing } \\
-\left(-\epsilon\left(c_{j} a_{i L}^{j-1}\right)\right) & c_{j} \text { positive crossing }
\end{array}\right\}=\epsilon\left(c_{j} a_{i L}^{j-1}\right) .
$$

$(k, \ell)=(i, i+1):$

(1) According to Figure 12, set $\epsilon^{\prime}\left(e_{2}\right)=\left(\epsilon\left(c_{j}\right)\right)^{-1}$ and so $\epsilon\left(b_{i+1, i}^{j}\right)=\left(\epsilon\left(c_{j}\right)\right)^{-1}$.

(2) As before, $\epsilon\left(a_{i+1, i}^{j}\right)=\epsilon^{\prime}\left(v_{i+1, i}\right)=\epsilon^{\prime}(0)=0$.

(3) We do have one correction: the disk $a_{i+1, i}^{j} a_{i L}^{j}$ in $\partial a_{i+1, L}^{j}$. Lemma 2.16 tells us

$$
\begin{aligned}
\epsilon\left(a_{i+1, L}^{j}\right) & = \begin{cases}\epsilon^{\prime}\left(a_{i+1, L}^{j}\right)-(-1)^{\left|t_{\alpha}^{ \pm 1}\right|} \epsilon\left(a_{i+1, L}^{j} ; a_{i+1, i}^{j} a_{i L}^{j}\right) \epsilon(t) \pm 1 \\
\epsilon^{\prime}\left(a_{i+1, L}^{j} b_{i+1, i}^{j} a_{i L}^{j}\right) & c_{j} \text { negative crossing }\end{cases} \\
& \begin{cases}\epsilon\left(a_{i L}^{j-1}\right)+\epsilon\left(t_{\alpha}^{ \pm 1} b_{i+1, i}^{j} a_{i L}^{j}\right) & c_{j} \text { negative crossing } \\
\epsilon\left(a_{i L}^{j-1}\right)-\epsilon\left(b_{i+1, i}^{j} a_{i L}^{j}\right) & c_{j} \text { positive crossing }\end{cases} \\
& =0,
\end{aligned}
$$

since

$$
\epsilon\left(b_{i+1, i}^{j} a_{i L}^{j}\right)=\left(\epsilon\left(c_{j}\right)\right)^{-1} \epsilon\left(c_{j} a_{i L}^{j-1}\right)=\epsilon\left(a_{i L}^{j-1}\right) .
$$

(4) As $\epsilon\left(a_{i+1, i}^{j}\right)=0$, no corrections are needed when moving the base point $*_{\alpha}$ over $a_{i+1, i}^{j}$. 
If $\epsilon^{\prime}$ is a $\rho$-graded augmentation, then $\rho|| c \mid$ for all augmented crossings $c$. We see that $\left|b_{i+1, i}^{j}\right|=\mu(i+1)-$ $\mu(i)=\left|c_{j}\right|$ and, since $\partial$ lowers degree by one,

$$
\begin{aligned}
\left|b_{L K}^{j}\right| & =\mu(L)-\mu(K) \\
& =\mu(L)-\mu(i)+\mu(i)-\mu(i+1)+\mu(i+1)-\mu(K) \\
& =-\left|a_{i L}^{j-1}\right|-\left|c_{j}\right|+\left|a_{i+1, K}^{j-1}\right|, \\
\left|a_{i+1, K}^{j}\right| & =\left|b_{i+1, K}^{j}\right|-1=\left|a_{i+1, K}^{j-1}\right|, \\
\left|a_{i L}^{j}\right| & =\left|b_{i L}^{j}\right|-1=\left|a_{i L}^{j-1}\right| .
\end{aligned}
$$

Since $\epsilon^{\prime}$ satisfies Property (R) on the $a^{j-1}$-lattice, we know $\epsilon$ is a $\rho$-graded augmentation which satisfies Property (R). In fact, $\epsilon$ is just $a_{i+1, i}^{j}, a_{i L}^{j}$ augmented with the rest of the augmentation on the $a^{j}$-lattice transferred from the $a^{j-1}$-lattice.

Configuration (c), (d), (e), (f): Similarly, one can extend $\epsilon_{j}$ over a crossing $c_{j}$ with the ruling having configuration (c), (d), (e), or (f) near $c_{j}$ to an augmentation $\epsilon_{j+1}$ satisfying Property (R) by defining it on new crossings as specified in Figure 12, We omit the calculations.

3.3. Right cusps. By construction and Lemma 2.16] $\epsilon=\epsilon_{n}$ is an augmentation. In this section, we will show that we do in fact have a ruling. Recall that $q_{1}, \ldots, q_{m}$ are the crossings at the right cusps numbered from top to top. Then

$$
\partial q_{k}=t_{k}^{ \pm 1}+a_{2 m-2 k+2,2 m-2 k+1}^{n}
$$

for $1 \leqslant k \leqslant m$, where the power of $t_{k}$ is determined by the orientation of the knot at the right cusp, since strands $2 m-2 k+2$ and $2 m-2 k+1$ are incident to the $k$-th right cusps from the bottom. Since $\epsilon$ is an augmentation,

$$
\begin{aligned}
0 & =\epsilon \partial q_{k} \\
& =\epsilon\left(t_{k}^{ \pm 1}+a_{2 m-2 k+2,2 m-2 k+1}^{n}\right) \\
& =\left(\epsilon\left(t_{k}\right)\right)^{ \pm 1}+\epsilon\left(a_{2 m-2 k+2,2 m-2 k+1}^{n}\right) .
\end{aligned}
$$

Since $0 \neq \epsilon(t)=\prod_{i=1}^{s} \epsilon\left(t_{i}\right)$,

$$
\epsilon\left(a_{2 m-2 k+2,2 m-2 k+1}^{n}\right)=-\left(\epsilon\left(t_{k}\right)\right)^{ \pm 1} \neq 0 .
$$

Since $\epsilon$ satisfies Property (R), this tells us strands $2 m-2 k+2$ and $2 m-2 k+1$ are paired at the right cusps for all $1 \leqslant k \leqslant m$ and so this construction does give a ruling.

This concludes the proof of the forward direction of Theorem 1.1. This construction also gives restrictions on $\epsilon(t)$ for any augmentation $\epsilon$. In particular, the final statement in Theorem 1.1

Theorem 3.1. If $\rho$ is even with $\rho \mid 2 r(K)$, then any $\rho$-graded augmentation $\epsilon$ satisfies $\epsilon(t)=-1$.

Proof. Consider the associated $\rho$-graded ruling. If $\rho$ is even, then any $\rho$-graded ruling is only switched at crossings $c_{k}$ with $\rho|| c_{k} \mid$ and so 2||$c_{k} \mid$. Thus any paired strands in the ruling have opposite orientation. If strand $i$ is oriented to the right, we assign that portion of the ruling, the sign +1 and if it is instead oriented to the left, we assign -1 . Define $\operatorname{sgn}(i, k)$ to be the sign for strands $i>j$ paired in the ruling between $c_{k}$ and $c_{k+1}$. Note that this sign can only change going over a switched crossing. 


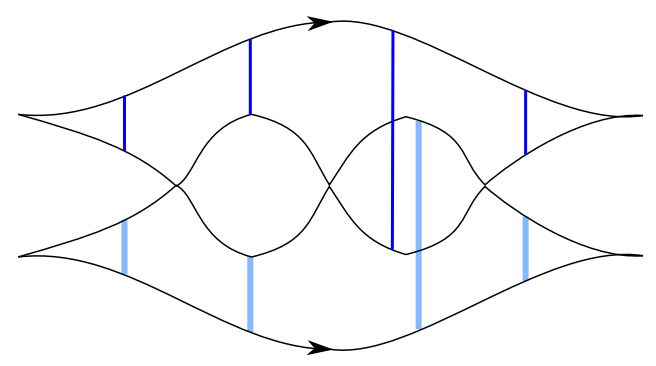

FIGURE 19. Oriented right handed trefoil with a normal graded ruling indicated.

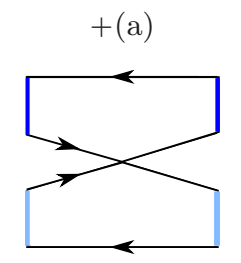

$\operatorname{sgn}(k, K)=-1$

$\operatorname{sgn}(k, i)=+1$ $+(\mathrm{a})$

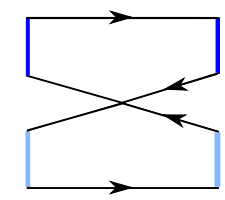

$\operatorname{sgn}(k, K)=+1 \quad$ s

$\operatorname{sgn}(k, i)=-1$ $+(\mathrm{b})$

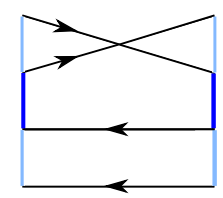

$\operatorname{sgn}(k, i)=+1$ $+(\mathrm{b})$

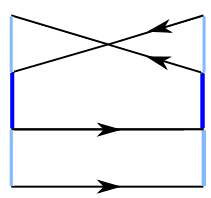

$\operatorname{sgn}(k, i)=-1$ $+(\mathrm{c})$

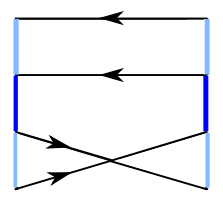

$\operatorname{sgn}(k, L)=-1$

$\operatorname{sgn}(k, K)=-1$

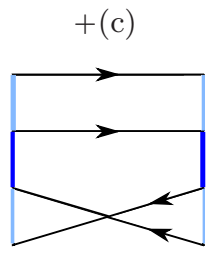

$\operatorname{sgn}(k, L)=+1$

$\operatorname{sgn}(k, K)=+1$

FIGURE 20. All possible ruling configurations and orientations near a crossing which is switched in a $\rho$-ruling when $\rho$ is even with signs of ruled pairs given with our strand labeling convention.

For example, if we have the trefoil with the orientation given in Figure 19 then

\begin{tabular}{l||cc|cc|cc|cc}
$k$ & 0 & 0 & 1 & 1 & 2 & 2 & 3 & 3 \\
\hline$i$ & 4 & 2 & 4 & 2 & 4 & 3 & 4 & 2 \\
\hline $\operatorname{sgn}(i, k)$ & +1 & -1 & +1 & -1 & +1 & -1 & +1 & -1
\end{tabular}

Given a $\rho$-graded ruling with $\rho$ even, we also see that we cannot have switched crossings which are negative crossings. So all switched crossings have one of the configurations appearing in Figure 20.

Note that in these switch configurations the signs of ruling pairs do not change. Thus, each ruling path is an oriented unknot. The important part of this is that if a ruling pair has sign +1 , respectively -1 , at the left cusp, then it has sign +1 , respectively -1 , at the right cusp.

We will show that for any $k$ such that $0 \leqslant k \leqslant n$,

$$
\prod\left(\epsilon\left(a_{r s}^{k}\right)\right)^{\operatorname{sgn}(r, k)}=1,
$$

where the product is taken over all paired strands $r$ and $s$ in the ruling between $c_{k}$ and $c_{k+1}$.

Clearly this is true for $k=0$. Induct on $k$. Suppose equation (3) is true for $k-1$. We will show that equation (3) holds for $k$. If the ruling is not switched at $c_{k}$, then the result is clear. If $c_{k}$ has configuration type $+(a)$, then, by Figure 12 ,

$$
\epsilon\left(a_{r s}^{k}\right)= \begin{cases}\epsilon\left(c_{k}\right) \epsilon\left(a_{K, i+1}^{k-1}\right) & (r, s)=(K, i+1) \\ \epsilon\left(c_{k}\right) \epsilon\left(a_{i L}^{k-1}\right) & (r, s)=(i, L) \\ \epsilon\left(a_{r s}^{k-1}\right) & \text { otherwise }\end{cases}
$$

and

$$
\operatorname{sgn}(K, k)=-\operatorname{sgn}(i, k), \quad \operatorname{sgn}(r, k)=\operatorname{sgn}(r, k-1)
$$


for all strands $r$ and $s$ paired in the ruling between $c_{k}$ and $c_{k+1}$. Thus

$$
\begin{aligned}
\prod_{r, s}\left(\epsilon\left(a_{r s}^{k}\right)\right)^{\operatorname{sgn}(r, k)} & =\left(\epsilon\left(c_{k}\right) \epsilon\left(a_{K, i+1}^{k-1}\right)\right)^{\operatorname{sgn}(K, k)}\left(\epsilon\left(c_{k}\right) \epsilon\left(a_{i L}^{k-1}\right)\right)^{\operatorname{sgn}(i, k)} \prod_{(r, s) \neq(K, i+1),(i, L)}\left(\epsilon\left(a_{r s}^{k-1}\right)\right)^{\operatorname{sgn}(r, k)} \\
& =\left(\epsilon\left(c_{k}\right)\right)^{-\operatorname{sgn}(i, k)}\left(\epsilon\left(a_{K, i+1}^{k-1}\right)\right)^{\operatorname{sgn}(K, k)}\left(\epsilon\left(c_{k}\right)\right)^{\operatorname{sgn}(i, k)}\left(\epsilon\left(a_{i L}^{k-1}\right)\right)^{\operatorname{sgn}(i, k)} \prod_{(r, s) \neq(K, i+1),(i, L)}\left(\epsilon\left(a_{r s}^{k-1}\right)\right)^{\operatorname{sgn}(r, k-1)} \\
& =\left(\epsilon\left(a_{K, i+1}^{k-1}\right)\right)^{\operatorname{sgn}(K, k-1)}\left(\epsilon\left(a_{i L}^{k-1}\right)\right)^{\operatorname{sgn}(i, k-1)} \prod_{(r, s) \neq(K, i+1),(i, L)}\left(\epsilon\left(a_{r s}^{k-1}\right)\right)^{\operatorname{sgn}(r, k-1)} \\
& =\prod_{r, s}\left(\epsilon\left(a_{r s}^{k-1}\right)\right)^{\operatorname{sgn}(r, k-1)} \\
& =1 .
\end{aligned}
$$

Similarly, we can see the same is true if $c_{k}$ has configuration $+(b)$ or $+(c) \operatorname{since} \operatorname{sgn}(r, k)=\operatorname{sgn}(r, k-1)$ for all strands $r$ and $s$ which are paired in the ruling between $c_{k}$ and $c_{k+1}$.

In particular, the result is true for $k=n$. Since

$$
\partial q_{\ell}=t_{\ell}^{\mathrm{sgn}(2 m-2 \ell+2,2 m-2 \ell+1)}+a_{2 m-2 \ell+2,2 m-2 \ell+1}^{n},
$$

we know

$$
0=\epsilon \partial q_{\ell}=\left(\epsilon\left(t_{\ell}\right)\right)^{\operatorname{sgn}(2 m-2 \ell+2, n)}+\epsilon\left(a_{2 m-2 \ell+2,2 m-2 \ell+1}^{n}\right)
$$

for all $1 \leqslant \ell \leqslant m$. Thus

$$
\epsilon\left(t_{\ell}\right)=-\left(\epsilon\left(a_{2 m-2 \ell+2,2 m-2 \ell+1}^{n}\right)\right)^{\operatorname{sgn}(2 m-2 \ell+2, n)}
$$

and so, if $s$ is the number of base points, then

$$
\begin{aligned}
\epsilon(t) & =\prod_{\ell=1}^{s} \epsilon\left(t_{\ell}\right)=(-1)^{s-m} \prod_{\ell=1}^{m}\left(-\left(\epsilon\left(a_{2 m-2 \ell+2,2 m-2 \ell+1}^{n}\right)\right)^{\operatorname{sgn}(2 m-2 \ell+2, n)}\right) \\
& =(-1)^{s} \prod_{\ell=1}^{m}\left(\epsilon\left(a_{2 m-2 \ell+2,2 m-2 \ell+1}^{n}\right)\right)^{\operatorname{sgn}(2 m-2 \ell+2, n)} \\
& =(-1)^{s} \\
& =-1
\end{aligned}
$$

as by Lemma 3.2 we know we have an odd number of base points.

Recall that we add an even number of base points if a crossing $c_{k}$ has configuration (d), (e), (f), or not augmented, two for each -(a) crossing, an odd number for each $+(\mathrm{a}), \pm(\mathrm{b}), \pm(\mathrm{c})$, and one for each right cusp. Thus, to show there are an odd number of base points, it suffices to show the following: (The following argument was communicated to the author by Lenhard Ng.)

Lemma 3.2. If $c$ gives the number of right cusps, $s$ is the number of switches in the ruling, and $a_{-}$is the number of -(a) crossings, then

$$
c+s+a_{-} \equiv 1 \bmod 2 .
$$




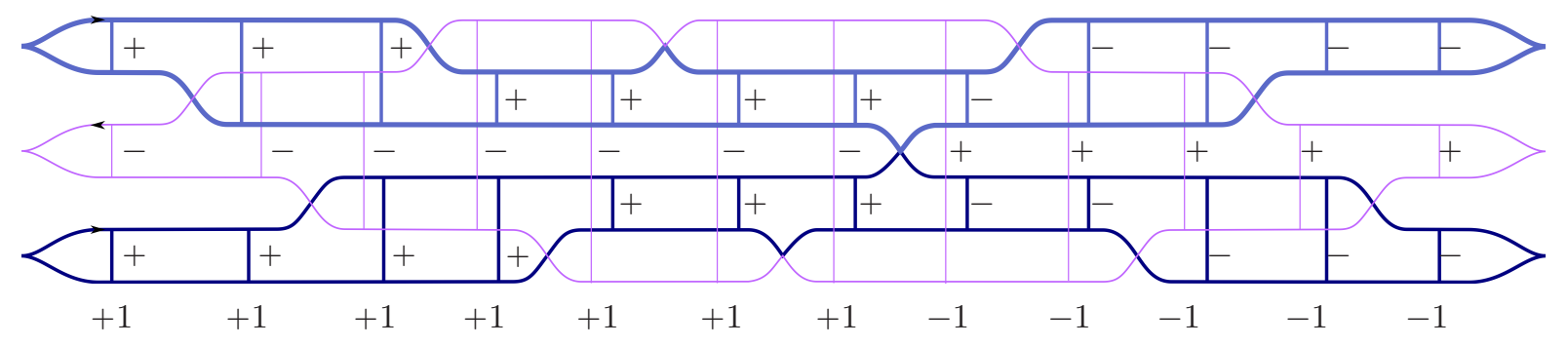

FiguRE 21. The diagram gives a normal ruling of the left handed trefoil. At each vertical slice of the diagram, the paired strands in the ruling are decorated with $+/-$, denoting the assignment of $+1 /-1$ to the corresponding paired strands. The number below the vertical slice gives the assigned sum over the ruled pairs.

Proof. We will prove this result by showing each of the following statements:

$$
\begin{aligned}
& t b+r \equiv \# \text { components } \bmod 2 \\
& t b \equiv c+c r \quad \bmod 2 \\
& c r \equiv s \quad \bmod 2 \\
& r \equiv a_{-} \quad \bmod 2
\end{aligned}
$$

where $r$ is the rotation number and $c r$ is the number of crossings. Note that if we add these four equations together, we get that

$$
c+s+a_{-} \equiv \# \text { components } \bmod 2 .
$$

Since in our case we have a knot, this gives the desired result.

Statement 4 is a standard result. Statement 5 results from the fact that the Thurston-Bennequin number is the number of right cusps plus the number of crossings counted with sign. To prove statement 6 , we will count the number of interlaced pairs from left to right.

We say that two pairs of points are interlaced if we encounter the pairs alternately as we move vertically. In other words, if $a_{i}$ denotes one pair of companion strands and $b_{i}$ denotes the other, then they appear from top to bottom as $a_{1} b_{1} a_{2} b_{2}$.

Note that the number of interlaced pairs does not change as we go from left to right over a switched crossing and changes by \pm 1 as we go from left to right over a nonswitched crossing. We also know that we have zero interlaced pairs at the left and right cusps. Thus, the number of nonswitched crossings, which is equal to the number of crossings minus the number of switched crossings, is even, which gives

$$
c r \equiv s \quad \bmod 2 .
$$

The proof of statement 7 will be a little more involved. First, at any vertical segment of the dipped diagram which does not include a crossing, if $r$ and $s(r>s)$ are paired, assign the pair the number 0 if they are oriented the same way and $\operatorname{sgn}(r, k)$ as defined in Theorem 3.1 otherwise. To any such vertical slice of the diagram, associate the sum of these numbers over the ruled pairs in that slice. For example, Figure 21 gives the assignments for the given ruling of the left handed trefoil.

One can check that this count goes up by \pm 2 as you go over a $-(a)$ crossing and otherwise does not change. At the left cusps, we compute the sum to be $u_{L}-d_{L}$, where $u_{L}$ is the number of up cusps and $d_{L}$ the number of down. At the right cusps, we compute the sum to be $d_{R}-u_{R}$, where $u_{R}$ and $d_{R}$ are defined 
analogously. Therefore we have

$$
\left(d_{R}-u_{R}\right) \equiv\left(u_{L}-d_{L}\right)+2 a_{-} \bmod 4
$$

So

$$
2 r \equiv 2 a_{-} \bmod 4
$$

and thus

$$
r \equiv a_{-} \bmod 2
$$

The augmentation variety is more complicated when $\rho$ is odd. Given a $\rho$-graded augmentation to a field $F$, once again, consider the associated $\rho$-graded ruling.

Remark 3.3. Looking at the various configurations for the switched crossings (see Figure 12), we see that for all paired strands $r, s$ between $c_{k}$ and $c_{k+1}$ with $r>s$,

$$
\epsilon\left(a_{r s}^{k}\right)=x^{2}
$$

for some $x \in F$ with $x \neq 0$. As before, since

$$
\partial q_{k}=t_{k}^{ \pm 1}+a_{2 m-2 k+2,2 m-2 k+1}^{n}
$$

we know

$$
\epsilon\left(t_{k}\right)=-\left(\epsilon\left(a_{2 m-2 k+2,2 m-2 k+1}^{n}\right)\right)^{ \pm 1}=-x^{2}
$$

for some $x \in F$ with $x \neq 0$. It is then clear that, if $s$ is the number of base points, then

$$
\epsilon(t)=\prod_{k=1}^{s} \epsilon\left(t_{k}\right)=(-1)^{s-m} \prod_{k=1}^{m} \epsilon\left(t_{k}\right)=(-1)^{s} x^{2}=-x^{2}
$$

for some $x \in F$ with $x \neq 0$ since, by Lemma 3.2, we know that $s$ is odd.

The following theorem, restated from the introduction, gives a slightly more precise result for when there exists a $\rho$-graded normal ruling for the diagram which is not oriented, meaning a ruling for which not all ruling strands are oriented unknots.

Theorem 1.2, If $\rho$ is odd and $\rho \mid 2 r(\Lambda)$, then

$$
\operatorname{Aug}_{\rho}(\Lambda)= \begin{cases}\left\{-x^{2}: x \in F^{*}\right\} & \text { if there exists a } \rho \text {-graded normal ruling of } \Lambda \text { which is not oriented } \\ \{-1\} & \text { if there exists a } \rho \text {-graded normal ruling of } \Lambda \text { and all rulings are oriented } \\ \varnothing & \text { if there are no } \rho \text {-graded normal rulings of } \Lambda .\end{cases}
$$

Proof. Suppose there exists a $\rho$-graded normal ruling for $\Lambda$ which is not oriented. Fix $0 \neq x \in F$. Since every ruling is oriented on the portion at the left cusps, for it to be an unoriented ruling, there has to be a crossing which takes the ruling from oriented to unoriented going from left to right. The only configurations for the ruling which do this are the crossings with configuration -(a), -(b), or -(c). Thus, a normal ruling of $\Lambda$ is not oriented if and only if it has a crossing with configuration -(a), -(b), or -(c). In fact, any ruling is also oriented at the right cusps and so must have at least two crossings where the ruling has configuration $-(\mathrm{a}),-(\mathrm{b})$, or $-(\mathrm{c})$.

Consider $\Lambda$ from the last crossing with configuration -(a),-(b), or $-(\mathrm{c})$, which we will denote $c_{k}$, to the right cusps. Note that any crossing with configuration $+(\mathrm{a}),+(\mathrm{b}),+(\mathrm{c}), \pm(\mathrm{d}), \pm(\mathrm{e}), \pm(\mathrm{f})$, or not switched preserves the orientation of the paired strands in the ruling. In other words, whatever orientation the strands 
in the ruling have just to the right $c_{k}$ is the orientation they have all the way through to the right cusps. Let $\sigma \in S_{2 m}$ be the permutation of the strands so that if strands $r$ and $s$ with $r>s$ are paired in the ruling immediately to the right of the crossing $c_{k}$, then strand $\sigma(r)$ is the strand with higher label and $\sigma(s)$ is the strand with lower label if we follow the ruled pair to the right cusps. (Note that $\sigma(r)=\sigma(s)+1$ and $2 \mid \sigma(r)$.)

As in the $\rho$ even case, set the orientation $\operatorname{sgn}(r, j)=1$ if strand $r$ is oriented to the right immediately after crossing $c_{j}$ and $\operatorname{sgn}(r, j)=-1$ if strand $r$ is oriented to the left for $k \leqslant j \leqslant n$. Labeling strands as before, this gives us

$$
\operatorname{sgn}(\max (i+1, K), k) \operatorname{sgn}(\max (i, L), k)= \begin{cases}+1 & \text { if } c_{k} \text { has configuration }-(\mathrm{a}) \\ -1 & \text { if } c_{k} \text { has configuration }-(\mathrm{b}) \text { or }-(\mathrm{c}) .\end{cases}
$$

Set $\ell_{r}=m+1-\frac{\sigma(r)}{2}$. Note that $\ell$ is chosen so that $\sigma(r)$ and $\sigma(s)$ are the strands crossing at $q_{\ell}$. Thus

$$
\partial q_{\ell_{r}}=t_{\ell_{r}}^{\operatorname{sgn}(r, k)}+a_{\sigma(r), \sigma(s)}^{n}
$$

and so

$$
\epsilon\left(t_{\ell_{r}}\right)=-\left(\epsilon\left(a_{\sigma(r), \sigma(s)}^{n}\right)\right)^{\operatorname{sgn}(r, k)}
$$

$\operatorname{since} \operatorname{sgn}(r, k)= \pm 1$.

Define $\epsilon$, an augmentation to $F$ of the DGA $\left(\mathcal{A}\left(\Lambda^{\prime}\right), \partial\right)$ of the dipped diagram $\Lambda^{\prime}$ of $\Lambda$, satisfying Property (R), by

$$
\epsilon\left(c_{j}\right)= \begin{cases}x^{\operatorname{sgn}(K, k)} & j=k \text { and } c_{j} \text { has configuration }-(\mathrm{a}),-(\mathrm{c}) \\ x^{\operatorname{sgn}(i, k)} & j=k \text { and } c_{j} \text { has configuration }-(\mathrm{b}) \\ 1 & \text { if the ruling is switched at } c_{j} \text { and } j \neq k \\ 0 & \text { otherwise. }\end{cases}
$$

Note that Property $(\mathrm{R})$ tells us that

$$
\epsilon\left(a_{r s}^{k}\right)=\epsilon\left(a_{\sigma(r), \sigma(s)}^{n}\right)
$$

for all strands $r$ and $s$ paired in the ruling between $c_{k}$ and $c_{k+1}$. We also note that $\epsilon$ must be a $\rho$-graded augmentation, since it was defined using a $\rho$-graded normal ruling.

We see that if $c_{j}$ has configuration $-(\mathrm{a})$, then

$$
\epsilon\left(a_{\sigma(r), \sigma(s)}^{n}\right)= \begin{cases}x^{\operatorname{sgn}(K, k)} & (r, s)=(K, i+1) \\ 1 & r, s \text { paired in ruling } \\ 0 & \text { otherwise. }\end{cases}
$$

If $c_{j}$ has configuration $-(\mathrm{b})$, then

$$
\epsilon\left(a_{\sigma(r), \sigma(s)}^{n}\right)= \begin{cases}x^{\operatorname{sgn}(i, k)} & (r, s)=(i, L) \\ x^{-\operatorname{sgn}(i, k)} & (r, s)=(i+1, K) \\ 1 & r, s \text { paired in ruling } \\ 0 & \text { otherwise. }\end{cases}
$$


Is $c_{j}$ has configuration $-(\mathrm{c})$, then

$$
\epsilon\left(a_{\sigma(r), \sigma(s)}^{n}\right)= \begin{cases}x^{\operatorname{sgn}(K, k)} & (r, s)=(K, i+1) \\ x^{-\operatorname{sgn}(K, k)} & (r, s)=(L, i) \\ 1 & r, s \text { paired in ruling } \\ 0 & \text { otherwise. }\end{cases}
$$

We know

$$
\begin{aligned}
& \epsilon(t)=\prod_{j=1}^{s} \epsilon\left(t_{j}\right)=(-1)^{s-m} \prod_{j=1}^{m} \epsilon\left(t_{m-j+1}\right) \\
& =(-1)^{s-m} \prod_{j=1}^{m}\left(-\left(\epsilon\left(a_{2 j, 2 j-1}^{n}\right)\right)^{\operatorname{sgn}(2 j, n)}\right) \\
& =(-1)^{s}\left(\epsilon\left(a_{\{\sigma(K), \sigma(i+1)\}}^{n}\right)\right)^{\operatorname{sgn}(\max (K, i+1), k)}\left(\epsilon\left(a_{\{\sigma(i), \sigma(L)\}}^{n}\right)\right)^{\operatorname{sgn}(\max (i, L), k)} \prod_{j=1}^{m}\left(\epsilon\left(a_{2 j, 2 j-1}^{n}\right)\right)^{\operatorname{sgn}(2 j, n)} \\
& j \neq m-\ell_{\max (K, i+1)}+1, m-\ell_{\max (L, i)}+1 \\
& =-\left(\epsilon\left(a_{\{\sigma(K), \sigma(i+1)\}}^{n}\right)\right)^{\operatorname{sgn}(\max (K, i+1), k)}\left(\epsilon\left(a_{\{\sigma(i), \sigma(L)\}}^{n}\right)\right)^{\operatorname{sgn}(\max (i, L), k)} \\
& = \begin{cases}-\left(x^{\operatorname{sgn}(K, k)}\right)^{\operatorname{sgn}(K, k)}\left(x^{\operatorname{sgn}(K, k)}\right)^{\operatorname{sgn}(i, k)} & c_{k} \text { has configuration - (a) } \\
-\left(x^{-\operatorname{sgn}(i, k)}\right)^{\operatorname{sgn}(i+1, k)}\left(x^{\operatorname{sgn}(i, k)}\right)^{\operatorname{sgn}(i, k)} & c_{k} \text { has configuration - (b) } \\
-\left(x^{\operatorname{sgn}(K, k)}\right)^{\operatorname{sgn}(K, k)}\left(x^{-\operatorname{sgn}(K, k)}\right)^{\operatorname{sgn}(L, k)} & c_{k} \text { has configuration - (c) }\end{cases} \\
& =-x^{2}
\end{aligned}
$$

by equation (8).

By Remark 3.3

$$
\operatorname{Aug}_{\rho}(\Lambda) \subset\left\{-x^{2}: x \in F^{*}\right\}
$$

so $\operatorname{Aug}_{\rho}(\Lambda)=\left\{-x^{2}: x \in F^{*}\right\}$.

Now suppose there exists a $\rho$-graded normal ruling for $\Lambda$ and all $\rho$-graded normal rulings of $\Lambda$ are oriented. In this case, the ruling must only have switched crossings with configuration $+(\mathrm{a}),+(\mathrm{b}),+(\mathrm{c}),(\mathrm{d}),(\mathrm{e})$, or (f). Note that the proof of Theorem 3.1 only required this be the case for the ruling, so the augmentation associated to the normal ruling must have $\epsilon(t)=-1$ and so $\operatorname{Aug}_{\rho}(\Lambda)=\{-1\}$.

If there do not exist any $\rho$-graded rulings for $\Lambda$, then clearly $\operatorname{Aug}_{\rho}(\Lambda)=\varnothing$.

\section{Ruling to Augmentation}

To show the backward direction of Theorem 1.1, that given a $\rho$-graded normal ruling of a front diagram of a Legendrian knot, we can find a $\rho$-graded augmentation of $\mathcal{A}_{R}$, it suffices to show that given a $\rho$-graded normal ruling of a front diagram, there exists a $\rho$-graded augmentation $\epsilon$ of the dipped diagram. We will do this by, in some sense, following the same argument as previously, but backwards. This includes the condition that the augmentation of the dipped diagram satisfies Property $(\mathrm{R})$. 
In particular, we will be able to find an augmentation $\epsilon$ of the dipped diagram satisfying Property (R) for which, if a crossing $c_{k}$ is augmented, $\epsilon\left(c_{k}\right)=1$ and such that $\epsilon\left(t_{1} \cdots t_{s}\right)=-1$ where $*_{1}, \ldots, *_{s}$ are the base points in the final diagram.

4.1. Definition of Augmentation. As previously, we can assume the base point $*$ corresponding to $t$ is in the loop of the top right cusp. We can then add one base point to each right cusp. We will set $\epsilon\left(t_{i}\right)=-1$ $(1 \leqslant i \leqslant m)$, this will also be true for the base points added subsequently. Note that we will not need to do any of the "correction" calculations for disks and base points as we are defining the map this way.

4.1.1. Left. For any ruling, at the left end of the diagram, we have strand $2 k$ paired with $2 k-1$ for $1 \leqslant k \leqslant m$, where $m$ is the number of right cusps. For $\epsilon$ to satisfy Property (R), we must set

$$
\epsilon\left(b_{r s}^{0}\right)=0
$$

for all $k$ and $\ell$ and

$$
\epsilon\left(a_{r s}^{0}\right)= \begin{cases}1 & \text { there exists } k \text { s.t. } r=2 k, s=2 k-1,1 \leqslant k \leqslant m \\ 0 & \text { otherwise. }\end{cases}
$$

4.1.2. Original crossings. Consider a crossing $c_{j}$. If the ruling is switched at $c_{j}$, set $\epsilon\left(c_{j}\right)=1$. If not, set $\epsilon\left(c_{j}\right)=0$. (Note that we can augment the switched crossings to any nonzero element of $F$ and still get an augmentation, but we may end up with an augmentation where $\epsilon(t) \neq-1$.)

Add base points and augment crossings in the dips, following Figure 12 .

4.2. Properties of the Augmentation. By the proof that augmentations imply rulings, $\epsilon$ is an augmentation and by the following, the resulting augmentation $\epsilon$ on the original undipped diagram with one base point $*$ associated to $t$ satisfies $\epsilon(t)=-1$.

Since we have set $\epsilon\left(t_{i}\right)=-1$ for all $1 \leqslant i \leqslant s$ and Lemma 3.2 tells us $s$ is odd,

$$
\epsilon(t)=\prod_{i=1}^{s} \epsilon\left(t_{i}\right)=(-1)^{s}=-1 .
$$

\section{Lifting Augmentations}

Given an augmentation to $\mathbb{Z} / 2$ of the Chekanov-Eliashberg DGA over $\mathbb{Z} / 2$. We will now use constructions similar to those in the proof of Theorem 1.1 to construct a lift of the augmentation to an augmentation to $\mathbb{Z}$ of the lift of the Chekanov-Eliashberg DGA and thus that one can construct an augmentation to any ring. Restating from the introduction:

Theorem 1.3. Let $\Lambda$ be a Legendrian knot in $\mathbb{R}^{3}$. Let $\left(\mathcal{A}_{\mathbb{Z} / 2}, \partial\right)$ be the Chekanov-Eliashberg DGA over $\mathbb{Z} / 2$ and let $\left(\mathcal{A}_{R}, \partial\right)$ be the $D G A$ over $R=\mathbb{Z}\left[t, t^{-1}\right]$. If $\epsilon^{\prime}: \mathcal{A}_{\mathbb{Z} / 2} \rightarrow \mathbb{Z} / 2$ is an augmentation of $\left(\mathcal{A}_{\mathbb{Z} / 2}, \partial\right)$, then one can find a lift of $\epsilon^{\prime}$ to an augmentation $\epsilon: \mathcal{A}_{R} \rightarrow \mathbb{Z}$ of $\left(\mathcal{A}_{R}, \partial\right)$ such that $\epsilon(t)=-1$.

Proof. Recall that $\mathcal{E}_{i}=\mathcal{A}\left(e_{1}, e_{2}\right)$ where $\left|e_{1}\right|=i-1,\left|e_{2}\right|=i, \partial\left(e_{2}\right)=e_{1}$, and $\partial\left(e_{1}\right)=0$ and $S_{i}\left(\mathcal{A}_{R}\left(a_{1}, \ldots, a_{n}\right)\right)=$ $\mathcal{A}_{R}\left(a_{1}, \ldots, a_{n}, e_{1}, e_{2}\right)$.

Note that, for any augmentation $\epsilon$ on $\mathcal{A}_{R}$ to $\mathbb{Z}$, there exists an augmentation $\hat{\epsilon}$ on $S\left(\mathcal{A}_{R}\right)$ to $\mathbb{Z}$ which agrees with $\epsilon$ on $\mathcal{A}_{R} \subset S\left(\mathcal{A}_{r}\right)$ and for any augmentation $\hat{\epsilon}$ on $S\left(\mathcal{A}_{R}\right)$ to $\mathbb{Z}$, there exists an augmentation $\epsilon$ on $\mathcal{A}_{R}$ to $\mathbb{Z}$ which agrees with $\epsilon$ on $\mathcal{A}_{R} \subset S\left(\mathcal{A}_{R}\right)$. And, we have the analogous result for any augmentation 
of $\mathcal{A}_{\mathbb{Z} / 2}$. Thus, clearly one can find a lift $\epsilon: \mathcal{A}_{R} \rightarrow \mathbb{Z}$ of $\epsilon^{\prime}: \mathcal{A}_{\mathbb{Z} / 2} \rightarrow \mathbb{Z} / 2$ if and only if one can find a lift $\epsilon: S\left(\mathcal{A}_{R}\right) \rightarrow \mathbb{Z}$ of $\epsilon: S\left(\mathcal{A}_{\mathbb{Z} / 2}\right) \rightarrow \mathbb{Z} / 2$.

So, if there exists a lift for $\mathcal{A}$, then there exists a lift for any stable tame isomorphic differential graded algebra. Therefore, to show the result, it suffices to show there exists a lift of the augmentation to $\mathbb{Z} / 2$ of differential graded algebras of knots in plat position. So we may assume $\Lambda$ is in plat position.

Given an augmentation $\epsilon^{\prime}: \mathcal{A}_{\mathbb{Z} / 2} \rightarrow F$ of the Chekanov-Eliashberg DGA over $\mathbb{Z} / 2$. Using Lemma 2.16 modulo 2 and the definition given in Figure $12 \bmod 2$, we can extend $\epsilon^{\prime}$ to an augmentation $\widehat{\epsilon}: \widehat{\mathcal{A}_{\mathbb{Z} / 2}} \rightarrow F$ of the DGA over $\mathbb{Z} / 2$ for the dipped diagram of $\Lambda$. We saw that if we know $\hat{\epsilon}\left(c_{i}\right)$ and the augmentation on the $a^{i} / b^{i}$-lattices for $i<j$, then

$$
\widehat{\epsilon}\left(c_{j}\right) \equiv \epsilon^{\prime}\left(c_{j}\right)+\sum_{i=0}^{j-1} \sum_{k, \ell} \sum_{p} \widehat{\epsilon}\left(Q_{p} b_{k \ell}^{i} R_{p}\right) \quad \bmod 2
$$

where, for $0 \leqslant i<j, \partial b_{k \ell}^{i}=P+\sum_{p} Q_{p} a_{k \ell}^{i} R_{p}$ before passing strand $k$ over strand $\ell$ in the creation of the dip between $c_{i}$ and $c_{i+1}$ and $P$ is the sum of terms which do not contain $a_{k \ell}^{i}$ with our labeling convention. This is the same as the construction introduced in [17. From [17] we know that this augmentation satisfies Property $(\mathrm{R})$.

Let $\left(\widetilde{\mathcal{A}_{Z}}, \widetilde{\partial}\right)$ be the lift of the Chekanov-Eliashberg DGA over $\mathbb{Z} / 2$ to a DGA over $Z=\mathbb{Z}\left[t_{1}^{ \pm 1}, \ldots, t_{s}^{ \pm 1}\right]$ of the DGA over $\mathbb{Z}\left[t_{1}^{ \pm 1}, \ldots, t_{s}^{ \pm 1}\right]$ of the dipped diagram of $\Lambda$. Define $\widetilde{\epsilon}: \widetilde{\mathcal{A}_{Z}} \rightarrow F$ by

$$
\widetilde{\epsilon}\left(c_{j}\right)= \begin{cases}1 & \text { if } \hat{\epsilon}\left(c_{j}\right) \neq 0 \\ 0 & \text { otherwise. }\end{cases}
$$

on the original crossings, define $\tilde{\epsilon}$ as given by Figure 12 for all other crossings, add base points where indicated in Figure 12, and define

$$
\widetilde{\epsilon}\left(t_{i}\right)= \begin{cases}-\widetilde{\epsilon}\left(a_{2 m-2 i+2,2 m-2 i+1}^{n}\right) & \text { if } 1 \leqslant i \leqslant m \\ -1 & \text { if } m<i \leqslant s .\end{cases}
$$

Note that all crossings and base points are augmented to 0 or \pm 1 . One can check that with this definition, $\tilde{\epsilon}$ is an augmentation of the dipped diagram of $\Lambda$. Note that as the same original crossings are augmented in the dipped diagram, this augmentation must correspond to the same ruling as $\widehat{\epsilon}$ and by definition, satisfies Property (R). So, clearly,

$$
\widetilde{\epsilon}(c) \equiv \widehat{\epsilon}(c) \quad \bmod 2
$$

for all crossings $c$ in the dipped diagram of $\Lambda$.

We will use induction on $k$ to show that

$$
\prod \tilde{\epsilon}\left(a_{p q}^{k}\right)=1
$$

where the product is taken over all paired strands $p$ and $q$, for all $1 \leqslant k \leqslant n$ and thus, that

$$
\prod_{i=1}^{s} \tilde{\epsilon}\left(t_{i}\right)=-1
$$

Since $\tilde{\epsilon}\left(a_{p q}^{0}\right)=1$ for $(p, q)=(2 m-2 k+2,2 m-2 k+1)$ for some $k$ such that $1 \leqslant k \leqslant m$, we know

$$
\prod_{p, q} \tilde{\epsilon}\left(a_{p q}^{0}\right)=1
$$


Looking at Figure 12, we see that

$$
\frac{\prod_{p, q} \tilde{\epsilon}\left(a_{p q}^{k}\right)}{\prod_{p, q} \widetilde{\epsilon}\left(a_{p q}^{k-1}\right)}=\left\{\begin{array}{ll}
\left(\widetilde{\epsilon}\left(c_{k-1}\right)\right)^{2} & \text { if the ruling has configuration (a) near } c_{k-1} \\
1 & \text { otherwise }
\end{array}\right\}=1,
$$

since $\tilde{\epsilon}\left(c_{k-1}\right)= \pm 1$. Thus, if $\prod \tilde{\epsilon}\left(a_{p q}^{k-1}\right)=1$, then $\prod \tilde{\epsilon}\left(a_{p q}^{k}\right)=1$. So, in particular, $\prod \tilde{\epsilon}\left(a_{p q}^{n}\right)=1$. Thus

$$
\begin{aligned}
\prod_{i=1}^{s} \tilde{\epsilon}\left(t_{i}\right) & =(-1)^{s-m} \prod_{i=1}^{m} \tilde{\epsilon}\left(t_{i}\right)=(-1)^{s-m} \prod_{i=1}^{m}\left(-\widetilde{\epsilon}\left(a_{2 m-2 i+2,2 m-2 i+1}^{n}\right)\right) \\
& =(-1)^{s} \prod_{i=1}^{m} \widetilde{\epsilon}\left(a_{2 m-2 i+2,2 m-2 i+1}^{n}\right)=(-1)^{s}=-1,
\end{aligned}
$$

since Lemma 3.2 tells $s$ is odd.

Lemma 2.16 in its original form also gives us a method to define an augmentation of the original diagram from an augmentation of the dipped diagram of $\Lambda$. Thus we have the augmentation $\epsilon: \mathcal{A}_{Z} \rightarrow F$ of the original diagram, defined by

$$
\epsilon\left(c_{j}\right)=\widetilde{\epsilon}\left(c_{j}\right)+\sum_{i=0}^{j-1} \sum_{k, \ell} \sum_{p} \epsilon\left(b_{k \ell}^{i} ; Q_{p}^{\prime} a_{k \ell}^{i} R_{p}^{\prime}\right)(-1)^{\left|\Phi\left(Q_{p}^{\prime}\right)\right|} \widetilde{\epsilon}\left(Q_{p}^{\prime} b_{k \ell}^{i} R_{p}^{\prime}\right)
$$

where, for $0 \leqslant i<j, \partial b_{k \ell}^{i}=P+\sum_{p} \epsilon\left(b_{k \ell}^{i} ; Q_{p}^{\prime} a_{k \ell}^{i} R_{p}^{\prime}\right) Q_{p}^{\prime} a_{k \ell}^{i} R_{p}^{\prime}$ before passing strand $k$ over strand $\ell$ in the creation of the dip between $c_{i}$ and $c_{i+1}$ and $P$ is the sum of terms which do not contain $a_{k \ell}^{i}$ with our labeling convention. Note that the "correction" disks in the $\mathbb{Z} / 2$ case are the same as the "correction" disks in the $\mathbb{Z}\left[t_{1}^{ \pm 1}, \ldots, t_{s}^{ \pm 1}\right]$ case, but the $\mathbb{Z}\left[t_{1}^{ \pm 1}, \ldots, t_{s}^{ \pm 1}\right]$ "correction" disks may be counted with negative sign and the disk may have extra corners at base points. Recall that $\widetilde{\epsilon}\left(t_{i}\right)=-1$ for $m<i \leqslant s$. Thus

$$
\widetilde{\epsilon}\left(Q_{p}^{\prime}\right) \equiv \widetilde{\epsilon}\left(Q_{p}\right) \bmod 2, \quad \widetilde{\epsilon}\left(R_{p}^{\prime}\right) \equiv \widetilde{\epsilon}\left(R_{p}\right) \bmod 2,
$$

since the disk which contributes $Q_{p}^{\prime}\left(\right.$ resp. $\left.R_{p}^{\prime}\right)$ to the differential may have extra corners at base points $t_{i}$ for $m<i \leqslant s$ (base points not occurring at right cusps) which the disk which contributes $Q_{p}$ (resp. $R_{p}$ ) to the differential does not have.

We will now show that $\epsilon$ is, in fact, a lift of $\epsilon^{\prime}$.

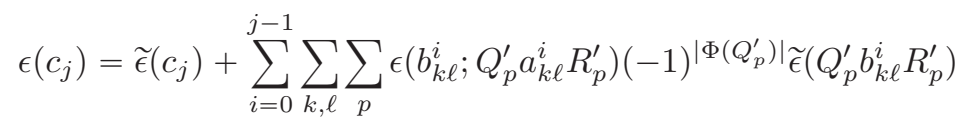

$$
\begin{aligned}
& \equiv \widetilde{\epsilon}\left(c_{j}\right)+\sum_{i=0}^{j-1} \sum_{k, \ell} \sum_{p} \tilde{\epsilon}\left(Q_{p} b_{k \ell}^{i} R_{p}\right) \bmod 2 \\
& \equiv\left(\epsilon^{\prime}\left(c_{j}\right)+\sum_{i=0}^{j-1} \sum_{k, \ell} \sum_{p} \hat{\epsilon}\left(Q_{p} b_{k \ell}^{i} R_{p}\right)\right)+\sum_{i=0}^{j-1} \sum_{k, \ell} \sum_{p} \tilde{\epsilon}\left(Q_{p} b_{k \ell}^{i} R_{p}\right) \quad \bmod 2 \\
& \equiv \epsilon^{\prime}\left(c_{j}\right)+2 \sum_{i=0}^{j-1} \sum_{k, \ell} \sum_{p} \hat{\epsilon}\left(Q_{p} b_{k \ell}^{i} R_{p}\right) \quad \bmod 2 \\
& \equiv \epsilon^{\prime}\left(c_{j}\right) \bmod 2 \text {, }
\end{aligned}
$$

since $\tilde{\epsilon}$ is a lift of $\hat{\epsilon}$. Note that this shows that the resulting augmentation of the DGA over $\mathbb{Z}\left[t_{1}^{ \pm 1}, \ldots, t_{s}^{ \pm 1}\right]$ is a lift and so, by the discussion of moving and adding base points in $\$ 2.5$, the augmentation of the DGA over $\mathbb{Z}\left[t, t^{-1}\right]$ is a lift as well, and

$$
\epsilon(t)=\prod_{i=1}^{s} \epsilon\left(t_{i}\right)=-1
$$


And, since $\mathbb{Z}$ embeds in any ring $R$, we can also use $\epsilon^{\prime}$ to define an augmentation $\epsilon: \mathcal{A}_{R} \rightarrow R$ with $\epsilon(t)=-1$.

\section{REFERENCES}

[1] Mina Aganagic, Tobias Ekholm, Lenhard Ng, and Cumrun Vafa. Topolocial strings, D-model, and knot contact homology. Preprint, 2013. http://arxiv.org/abs/1304.5778.

[2] Daniel Bennequin. Entrelacements et équations de Pfaff. In Third Schnepfenried geometry conference, Vol. 1 (Schnepfenried, 1982), volume 107 of Astérisque, pages 87-161. Soc. Math. France, Paris, 1983.

[3] Yu. V. Chekanov and P. E. Pushkar'. Combinatorics of fronts of Legendrian links, and Arnol'd's 4-conjectures. Uspekhi Mat. Nauk, 60(1(361)):99-154, 2005.

[4] Yuri Chekanov. Differential algebra of Legendrian links. Invent. Math., 150(3):441-483, 2002.

[5] Ya. M. Eliashberg. A theorem on the structure of wave fronts and its application in symplectic topology. Funktsional. Anal. i Prilozhen., 21(3):65-72, 96, 1987.

[6] Yakov Eliashberg. Invariants in contact topology. In Proceedings of the International Congress of Mathematicians, Vol. II (Berlin, 1998), number Extra Vol. II, pages 327-338, 1998.

[7] Yakov Eliashberg and Maia Fraser. Classification of topologically trivial Legendrian knots. In Geometry, topology, and dynamics (Montreal, PQ, 1995), volume 15 of CRM Proc. Lecture Notes, pages 17-51. Amer. Math. Soc., Providence, RI, 1998.

[8] John B. Etnyre. Legendrian and transversal knots. In Handbook of knot theory, pages 105-185. Elsevier B. V., Amsterdam, 2005.

[9] John B. Etnyre and Ko Honda. Knots and contact geometry. I. Torus knots and the figure eight knot. J. Symplectic Geom., 1(1):63-120, 2001.

[10] John B. Etnyre, Lenhard L. Ng, and Joshua M. Sabloff. Invariants of Legendrian knots and coherent orientations. J. Symplectic Geom., 1(2):321-367, 2002.

[11] Dmitry Fuchs. Chekanov-Eliashberg invariant of Legendrian knots: existence of augmentations. J. Geom. Phys., 47(1):4365, 2003.

[12] Dmitry Fuchs and Tigran Ishkhanov. Invariants of Legendrian knots and decompositions of front diagrams. Mosc. Math. J., 4(3):707-717, 783, 2004.

[13] Michael B. Henry and Dan Rutherford. Ruling polynomials and augmentations over finite fields. Preprint, 2013. http://arxiv.org/abs/1308.4662v1.

[14] Lenhard Ng. Framed knot contact homology. Duke Math. J., 141(2):365-406, 2008.

[15] Lenhard Ng and Daniel Rutherford. Satellites of Legendrian knots and representations of the Chekanov-Eliashberg algebra. Algebr. Geom. Topol., 13(5):3047-3097, 2013.

[16] Lenhard L. Ng. Computable Legendrian invariants. Topology, 42(1):55-82, 2003.

[17] Joshua M. Sabloff. Augmentations and rulings of Legendrian knots. Int. Math. Res. Not., (19):1157-1180, 2005.

Duke University, Durham, NC 27708

E-mail address: cleverso@math.duke.edu 